\title{
Bioactive-glass in Oral and Maxillofacial Surgery
}

\author{
Andrea Corrado Profeta, BDS (Hons), $\mathrm{PhD}^{1}$ Christoph Huppa, MD, DDS, PhD² \\ 1 Otto Schott Institute of Materials Research, Friedrich Schiller \\ University Jena, Jena, Germany \\ 2 Department of Oral and Maxillofacial Surgery, King's College Hospital \\ NHS Foundation Trust, Denmark Hill, London, United Kingdom

\begin{abstract}
Address for correspondence Andrea Corrado Profeta, BDS (Hons), $\mathrm{PhD}$, Otto Schott Institute of Materials Research, Friedrich Schiller University Jena, Fraunhoferstr. 6, 07743 Jena, Germany

(e-mail: andrea.profeta@uni-jena.de).
\end{abstract}

Craniomaxillofac Trauma Reconstruction 2016;9:1-14

\begin{abstract}
Keywords

- osteoplastic and reconstructive surgery

- alloplastic materials

- bioactive glasses

The use of synthetic materials to repair craniofacial defects is increasing today and will increase further in the future. Because of the complexity of the anatomy in the head and neck region, reconstruction and augmentation of this area pose a challenge to the surgeon. This review discusses key facts and applications of traditional reconstruction bone substitutes, also offering comparative information. It then describes the properties and clinical applications of bioactive-glass (B-G) and its variants in oral and maxillofacial surgery, and provides clinical findings. The discussion of each compound includes a description of its composition and structure, the advantages and shortcomings of the material, and its current uses in the field of osteoplastic and reconstructive surgery. With a better understanding of the available alloplastic implants, the surgeon can make a more informed decision as to which implant would be most suitable in a particular patient.
\end{abstract}

Successful repair of skull and facial defects, whether arising from trauma, tumor resection, or congenital disorders, continues to be a major challenge to oral and maxillofacial surgeons. Techniques vary, depending on the nature of the defect and quantity of tissue required. Reconstructions with autologous hard tissues have a long history with good clinical outcomes. ${ }^{1}$ However, bone autografts have certain disadvantages, such as limited availability when the volume of the defect is sizable, reduced dimensional stability together with an additional surgical site for the bone harvest, which prolongs operation time, convalescence, and donor-site morbidity. $^{2}$ Recently, several new alloplastic materials have expanded our arsenal of reconstructive options. Their benefits are not only the avoidance of donor-site morbidity but also the increasing availability and ready usage of biomaterials, three-dimensional stability, their cost effectiveness in reduction of the operating time, known composition and safety. $^{3}$ Nevertheless, a limited number of these products approach equivalence to autologous transplants. ${ }^{4}$ Failure of most implants originates at the interface between the biomaterial and its host tissue. ${ }^{5}$ This means that surface activity

received

September 8, 2014

accepted after revision

January 24, 2015

published online

May 21, 2016

of the alloplast is critical in evaluating its suitability for bone replacement, particularly if the area to be reconstructed will be subject to stress loading. Bioactive implants provide a potential solution to the problem of interface failure. ${ }^{5} \mathrm{~A}$ bioactive material is defined as one that elicits a specific biological response and forms a living bond with the host tissues, ${ }^{5}$ rather than forming an interfacial layer of scar tissue. This process prevents formation of a fibrous capsule surrounding the implant by the adhesion of repair tissues. Hench et $\mathrm{al}^{5}$ reported the first bioactive material in 1971 . They tested whether a phosphate containing silica-based material could fulfill the criterion of tissue bonding. The major breakthrough was that a degradable glass of the composition $\mathrm{SiO}_{2} 45, \mathrm{Na}_{2} \mathrm{O}$ 24.5, $\mathrm{CaO} 24.5$, and $\mathrm{P}_{2} \mathrm{O}_{5} 6$ in weight percent (wt\%), later termed $45 \mathrm{~S} 5$, formed a bond with living bone so strong that it could not be removed without breaking the bone. ${ }^{5}$ The selection of the composition was ideal; the low silica content made the glass easy to melt but also gave it the ability to form a surface layer of hydroxyapatite (HA) chemically and structurally similar to the mineral phase of human bone, following the immediate release of ionic species from the bulk material

Copyright $\odot 2016$ by Thieme Medical Publishers, Inc., 333 Seventh Avenue, New York, NY 10001, USA. Tel: +1(212) 584-4662.
DOI http://dx.doi.org/ 10.1055/s-0035-1551543. ISSN $1943-3875$. 


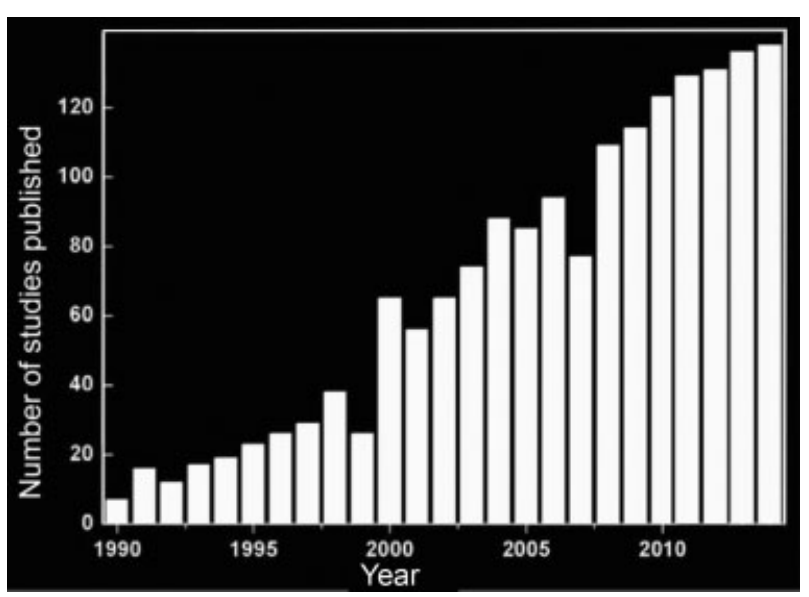

Fig. 1 Number of studies published per year in the field of biologically active glass ceramics ( $B-G$ and its variants). Compiled from a literature search in Web of Science performed in December 2014.

in contact with body fluids. ${ }^{6}$ It has been shown that the glass dissolves at a rate equal to that at which new host tissue is remodeled, serving as a biocompatible interface along which bone cells migrate due to its osteogenic properties. ${ }^{5}$ Notably, the constituents are physiological chemicals found in the body, typically silicium, sodium, potassium, magnesium, oxygen, calcium, and phosphorus. According to several studies, the concentration of these chemicals never rise to levels that could disturb the adjacent tissues. ${ }^{6,7}$ In this respect, biologically active glasses have equally demonstrated considerable antibacterial ${ }^{8}$ and angiogenesis-promoting characteristics. $^{9}$ The antimicrobial properties exhibited are likely multifactorial involving the creation of a local alkaline environment and the resistance of the material to bacterial adhesion and biofilm formation. ${ }^{10,11}$ Integration of mesenchymal stem cells and various growth factors such as vascular endothelial growth factor serve to augment the materials ability to repair tissue defects and stimulate neovascularization. $^{12}$ Hench's $45 \mathrm{~S} 5$ bioactive-glass (B-G), known also as Bioglass $^{\circledR}$, as it was trademarked by the University of Florida, and its variant products have been used in a variety of different shapes such as plates, rods, or rigid devices as well as in the form of particulate in many medical applications ( - Fig. 1). ${ }^{13-22}$ Clinically, these surface-reactive glassceramic biomaterials have been thoroughly investigated by oral and maxillofacial surgeons (-Table $\mathbf{1}$ ). This article reviews their use both in craniomaxillofacial trauma and bone reconstructive surgery, as well as in oral surgery, starting with the former.

\section{Calvarial Defects Reconstruction}

Calvarial bone reconstruction is adopted to repair large skull bone defects resulting from the treatment of tumors, infections, trauma, intracranial hemorrhage, or infarction. These defects cause discomfort as well as other functional problems to patients. Reconstructive surgery serves to protect the brain, provides a satisfying aesthetic outcome, and frequently involves the use of preserved allogenous bone grafts, as not enough autologous hard tissues are available. However, the inherent drawbacks of this approach strongly urge clinicians and researchers to explore alternative treatment options. ${ }^{23}$ Currently, several alloplastic materials, such as metals, plastics, ceramics, and fiber-reinforced composites (FRCs) are used for the reconstruction of skull bone defects under compromised healing conditions. ${ }^{24}$ Metallic implants have been used alone ${ }^{25}$ or combined with bone dust or HA cement to enhance bone ingrowth and integrity in cranial bone reconstructions. ${ }^{26}$ Polymethylmethacrylate (PMMA), polyethylene, and polyether ketone have been used in various formulations. ${ }^{27}$ Although acrylic resins are readily available for immediate usage and can be easily shaped either with manual or handpiece instruments during the operation, they do not attach chemically to surrounding bone and, later on, foreign-body reactions have been observed. ${ }^{27}$ In cranioplasty, late plate exposure and alloplast displacement, because of lack of incorporation at the donor site, are practical disadvantages associated with the use of these materials, in which case the removal becomes necessary with a technically challenging operation. ${ }^{27}$

Previous studies have reported on 45S5 B-G particles mixed with autologous bone particles harvested from cranial burr holes as an adjunct to cranial vault reconstruction. ${ }^{28,29}$ This was done to reconstruct full-thickness defects in two young patients, when minimal spontaneous bone regeneration was expected. On follow-up computer tomography (CT) scans, these patients demonstrated conversion of the majority of the reconstructed defect to bone density within 6 months. At 4-year follow-up, both patients had stable reconstruction, and there was no need for reoperation or biopsy of the biomaterial.

A promising new development was represented by the use of customized porous implants, made of a supporting $\mathrm{FRCs}^{23}$ or PMMA ${ }^{30}$ framework and modified B-G (S53P4), to combine workability of resinous materials with innate bioactive/antimicrobial properties of the latter in calvarial ${ }^{23}$ and midface bone $^{30}$ reconstructions. Beneficial effects of S53P4 particles with the composition $\mathrm{SiO}_{2} 53, \mathrm{Na}_{2} \mathrm{O} 23, \mathrm{CaO} 20$, and $\mathrm{P}_{2} \mathrm{O}_{5} 4.0$ $\mathrm{wt} \%$ have been demonstrated experimentally ${ }^{10,11}$ and put to use for various clinical indications. ${ }^{13,14,16,31-33}$ In the manufacturing process, prototyping models of the defect regions were created with additive manufacturing technology and these templates were used to prepare the patientspecific implants (- Fig. 2). ${ }^{23,30}$ The last mentioned were perforated with holes $1.5 \mathrm{~mm}$ in diameter to enhance body fluid perfusion, coated with S53P4 granules of 0.5 to $0.8 \mathrm{~mm}$ in size and the application of prostheses (overall average thickness of $3.5 \mathrm{~mm}$ ) was done with either biodegradable fixation screws or sutures. In longitudinal clinical and radiological examinations, with follow-up times up to 4 years ${ }^{23}$ and 5 years, ${ }^{30}$ normal progressive wound healing with diminishing postoperative clinical symptoms was observed and the implants retained their original position regardless of the fixation type, providing the expected functional as well as aesthetic outcome at all time points. Furthermore, there were no long-term complications such as implant-induced skull resorption, or later inflammatory reactions and acute 
Bioactive-glass in Oral and Maxillofacial Surgery Profeta, Huppa 3

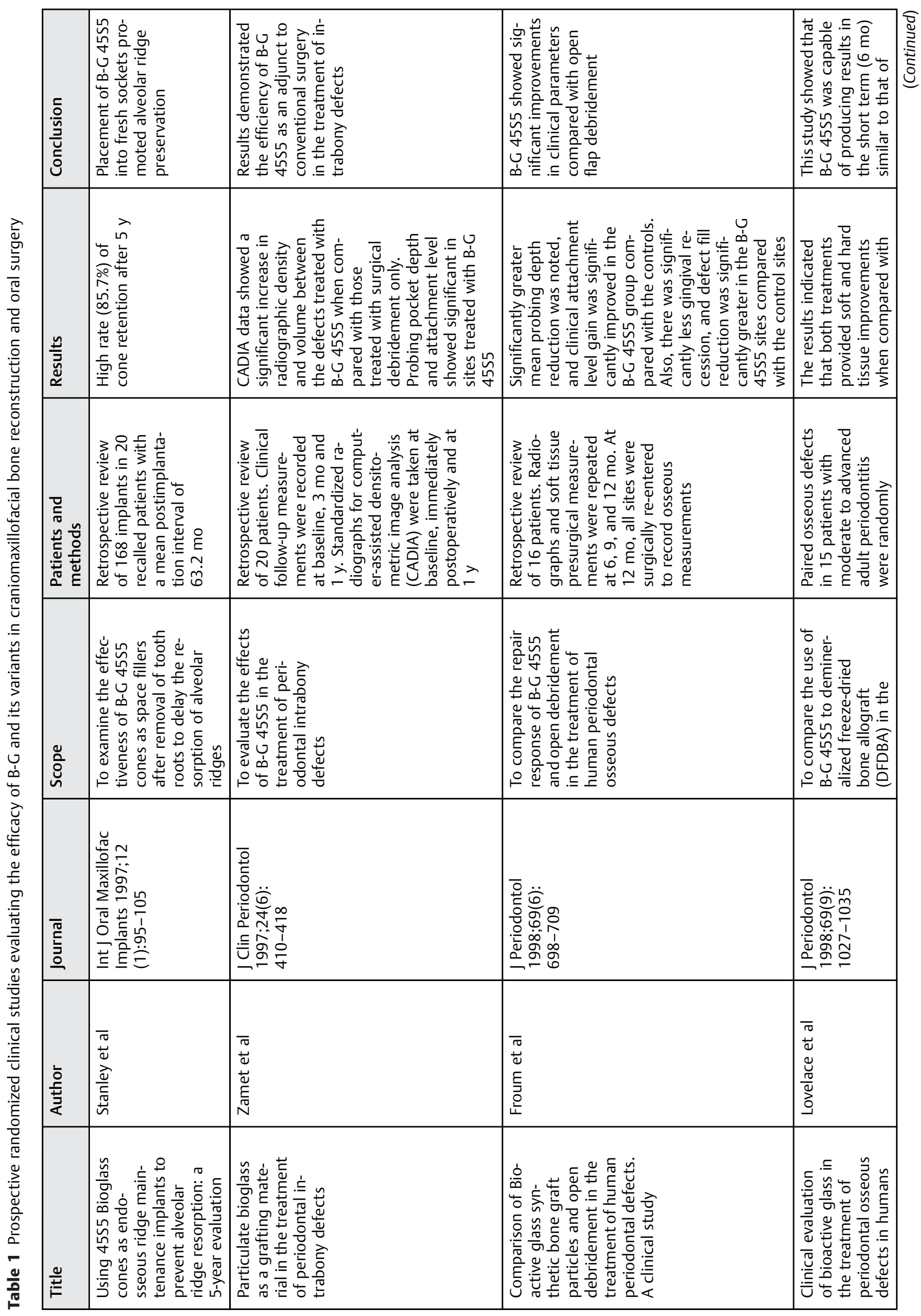




\begin{tabular}{|c|c|c|c|c|}
\hline 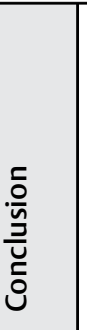 & 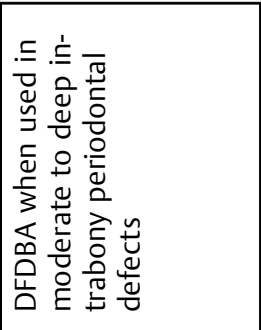 & 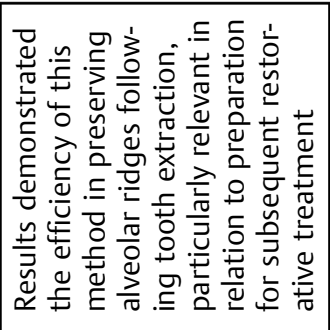 & 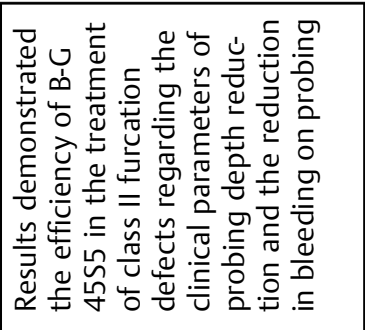 & 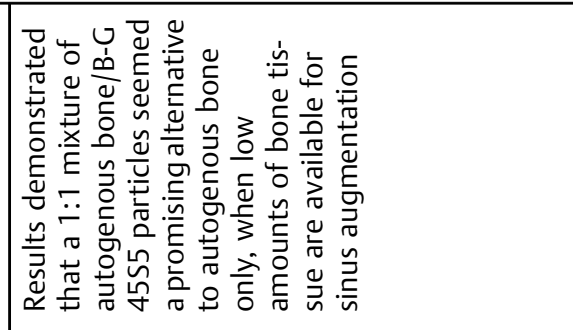 \\
\hline$\frac{\underline{n}}{\overrightarrow{\underline{Z}}}$ & 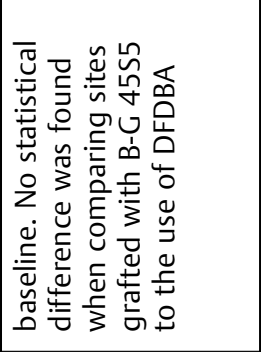 & 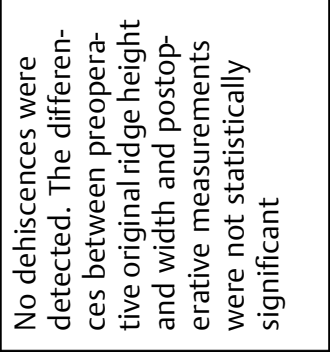 & 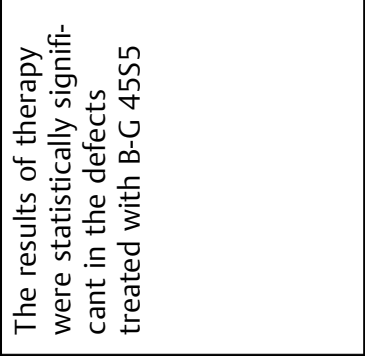 & 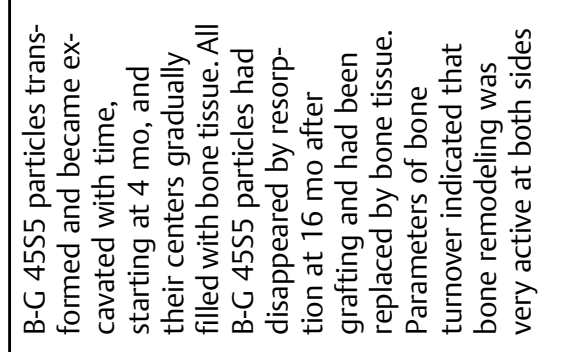 \\
\hline 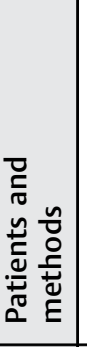 & 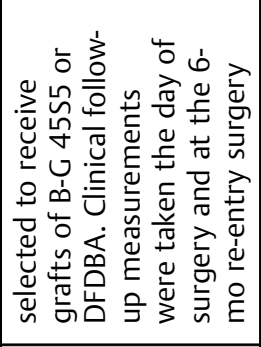 & 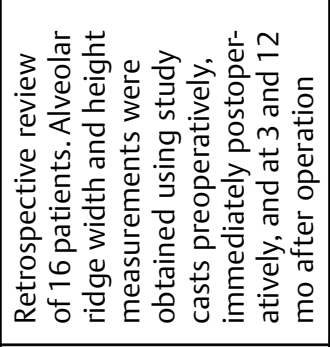 & 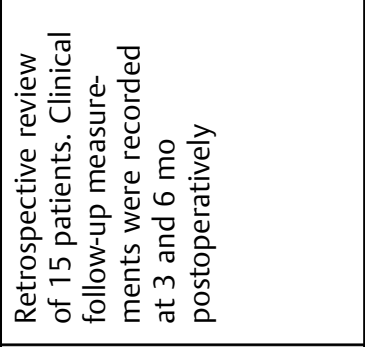 & 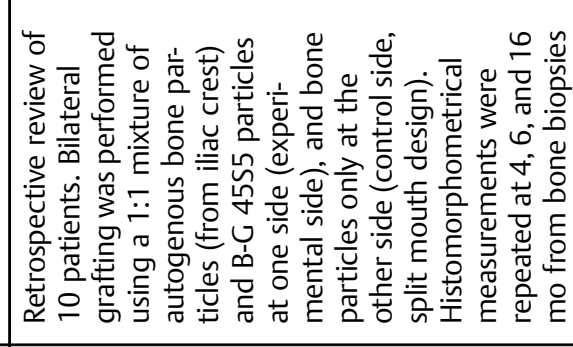 \\
\hline 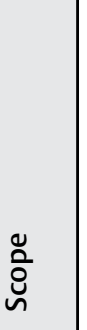 & 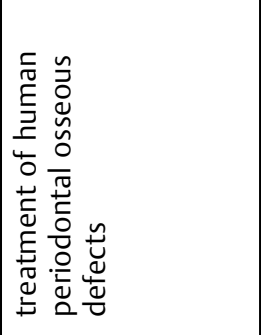 & 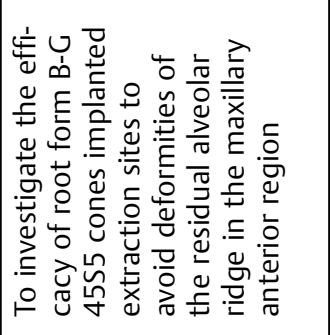 & 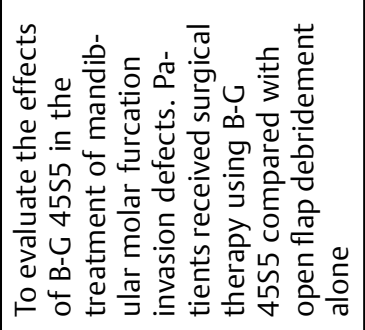 & 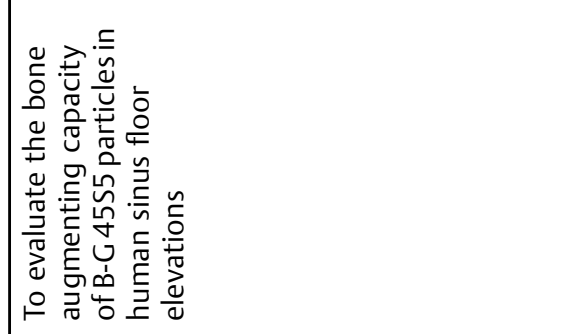 \\
\hline $\begin{array}{l}\overline{\widetilde{\sigma}} \\
\text { 总 }\end{array}$ & & 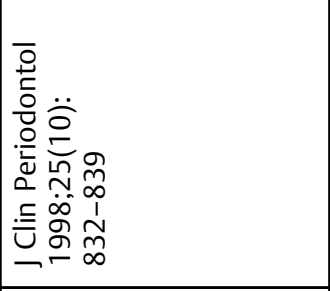 & 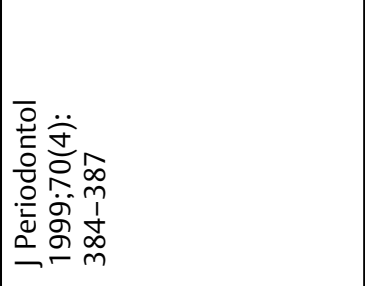 & 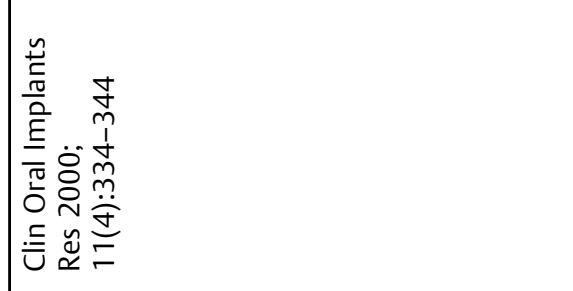 \\
\hline \begin{tabular}{l} 
哀 \\
\multirow{z}{*}{}
\end{tabular} & & 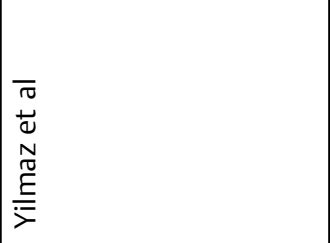 & 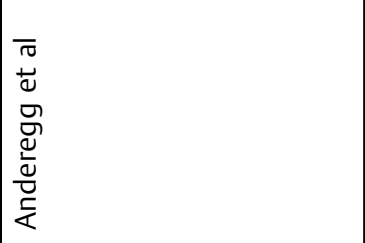 & 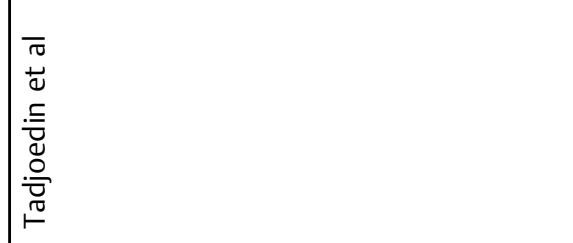 \\
\hline$\stackrel{\varrho}{\rightleftarrows}$ & & 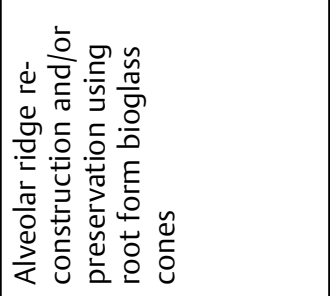 & 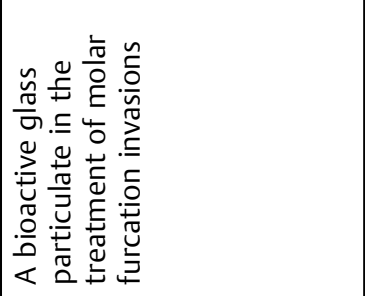 & 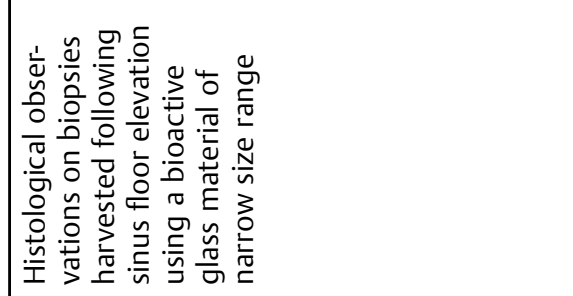 \\
\hline
\end{tabular}




\begin{tabular}{|c|c|c|c|c|}
\hline 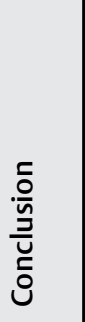 & 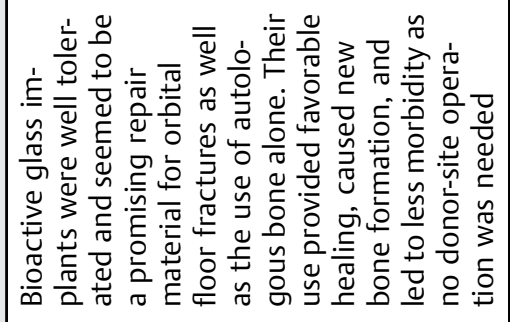 & 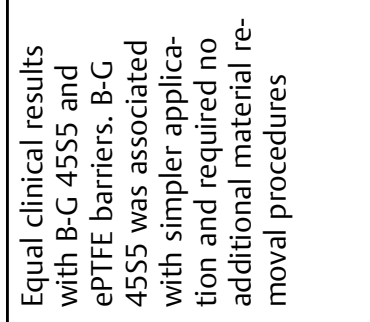 & 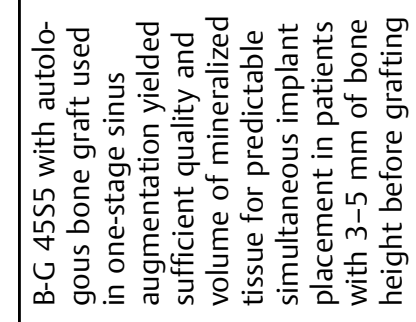 & 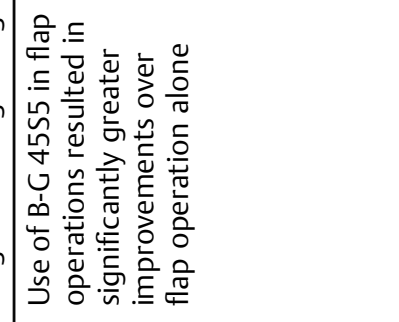 \\
\hline $\begin{array}{l}\frac{\underline{y}}{\vec{y}} \\
\stackrel{y}{u}\end{array}$ & 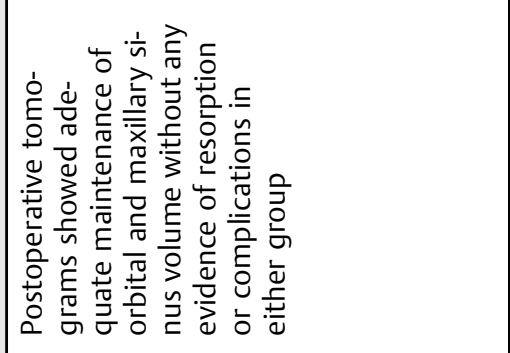 & 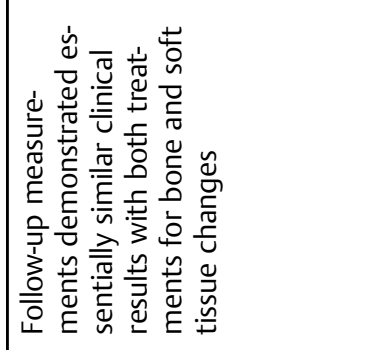 & 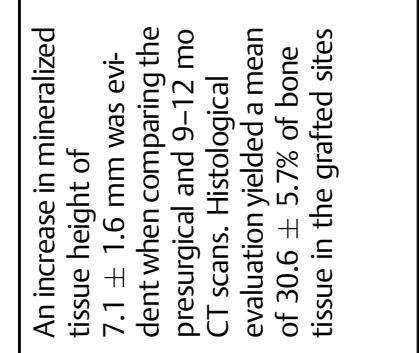 & 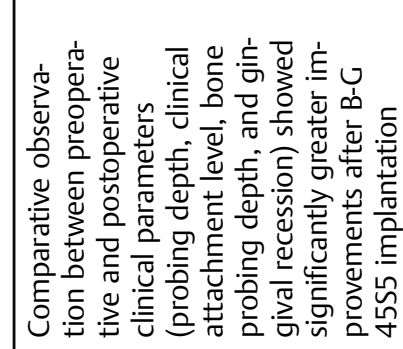 \\
\hline 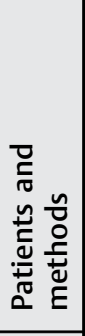 & 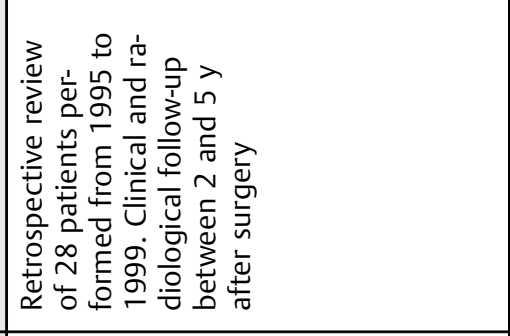 & 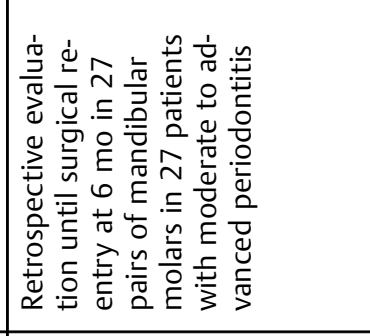 & 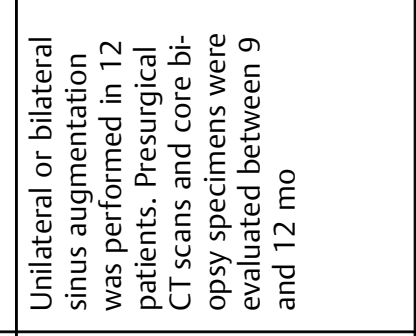 & 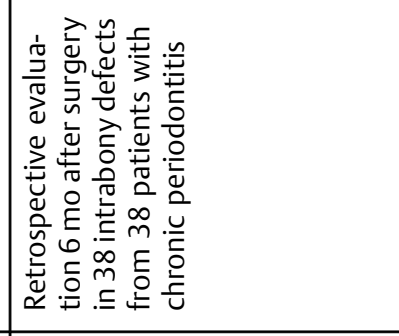 \\
\hline 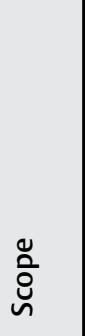 & 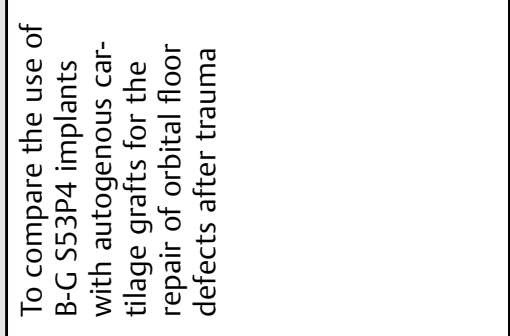 & 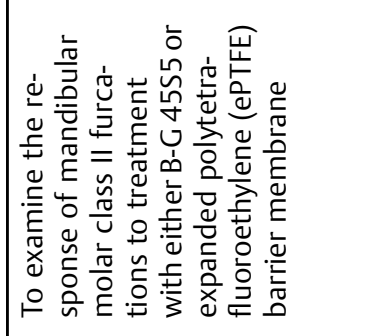 & 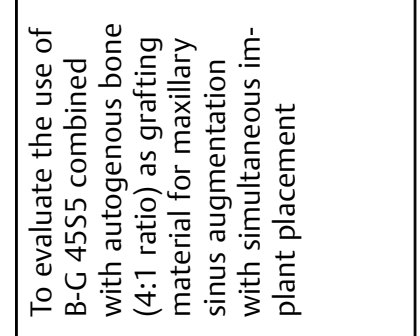 & 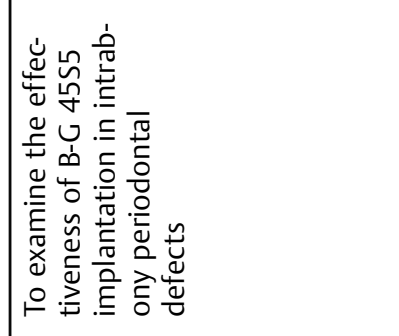 \\
\hline 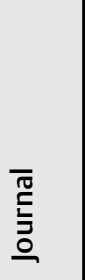 & 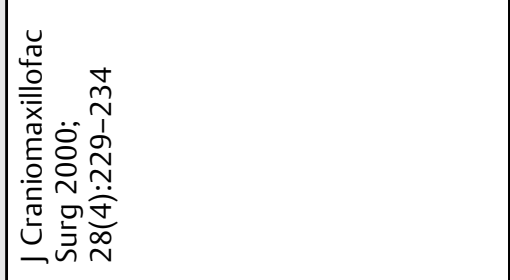 & 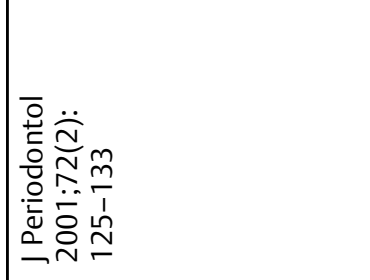 & 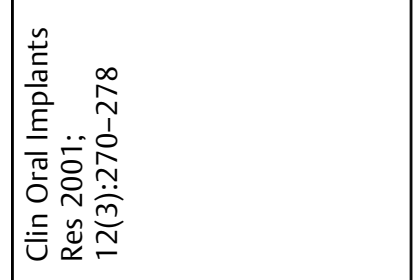 & 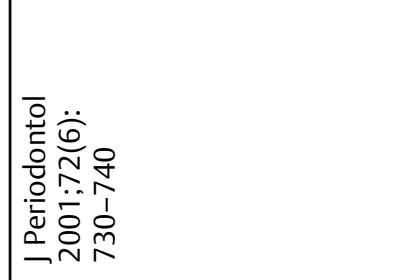 \\
\hline 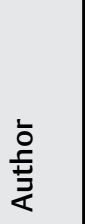 & 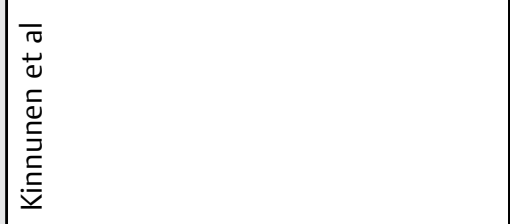 & 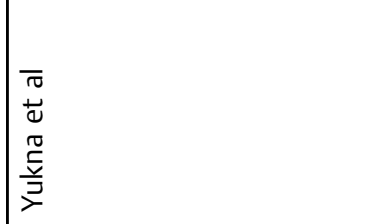 & 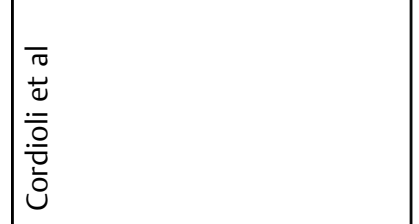 & 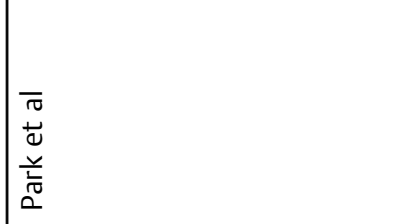 \\
\hline$\stackrel{\mathscr{D}}{\stackrel{\underline{H}}{*}}$ & 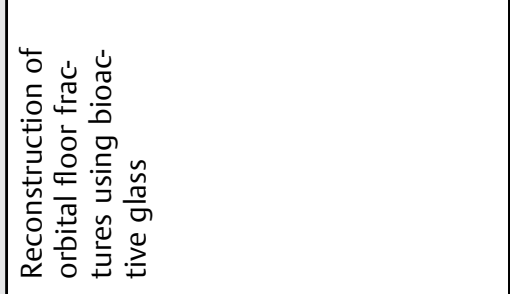 & 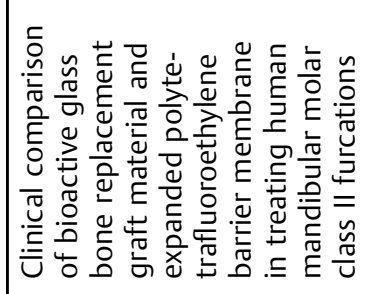 & 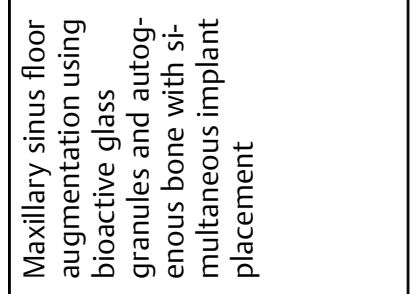 & 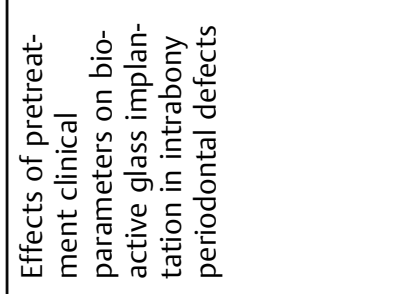 \\
\hline
\end{tabular}




\begin{tabular}{|c|c|c|c|c|}
\hline 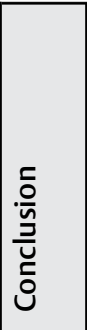 & 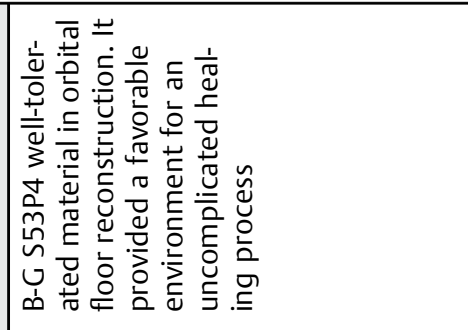 & 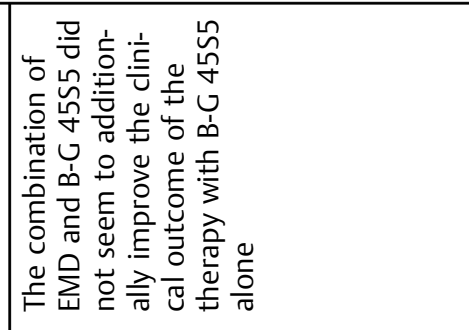 & 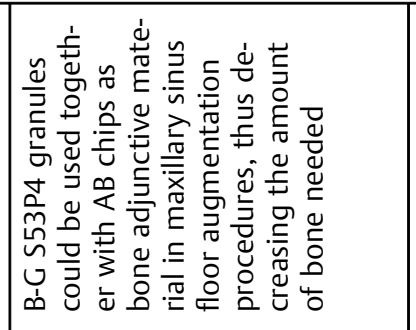 & 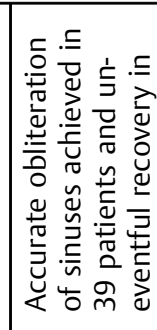 \\
\hline 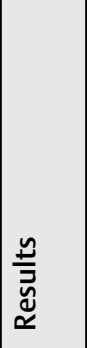 & 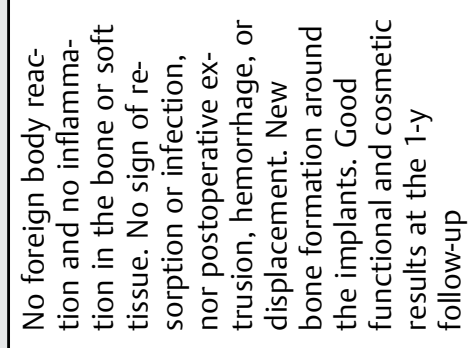 & 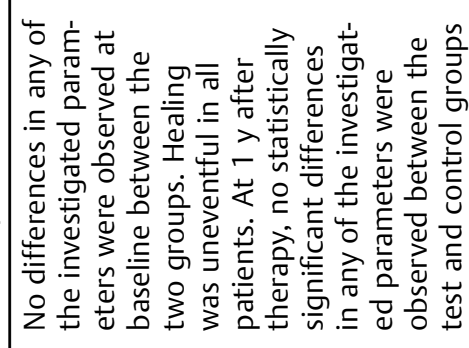 & 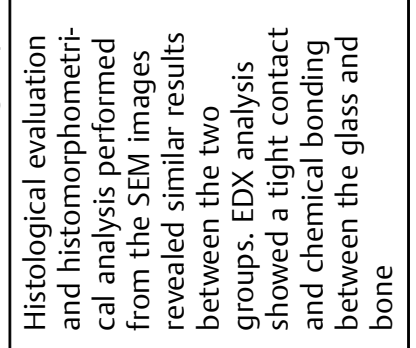 & 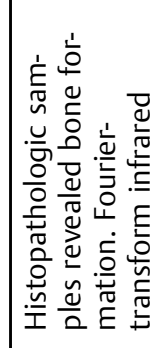 \\
\hline 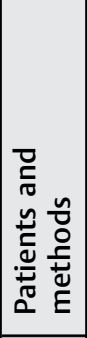 & 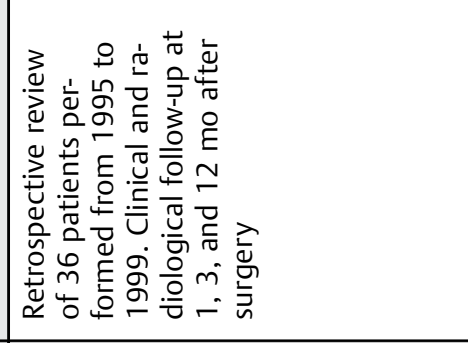 & 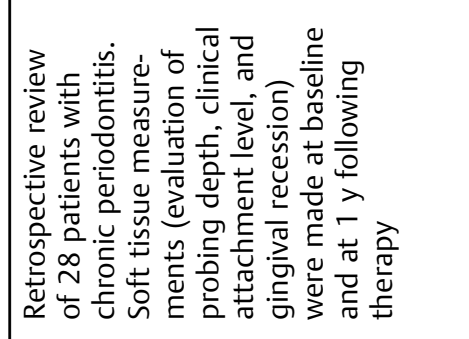 & 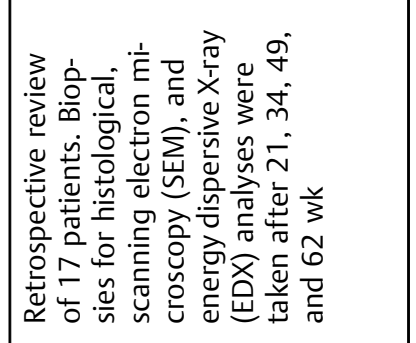 & 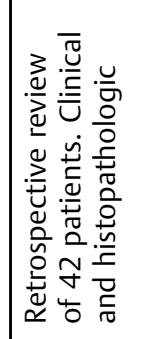 \\
\hline 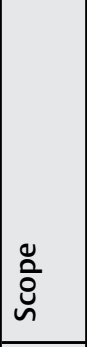 & 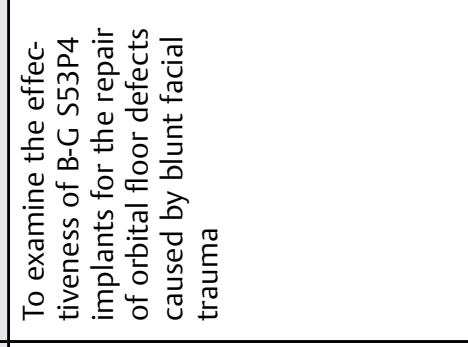 & 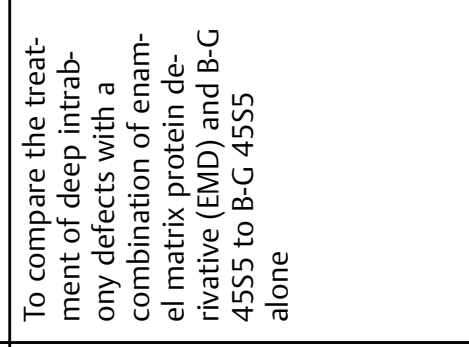 & 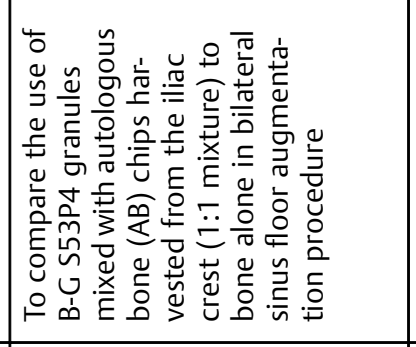 & 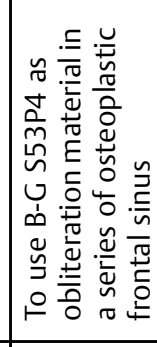 \\
\hline 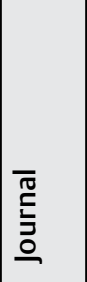 & 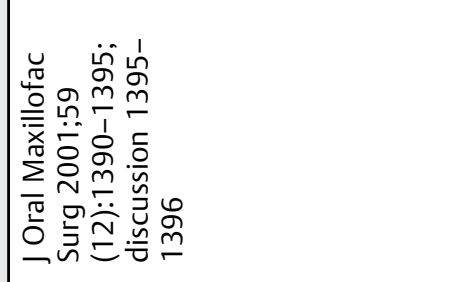 & 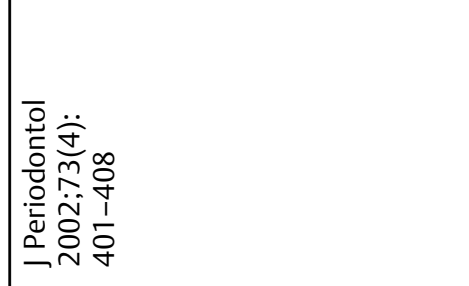 & 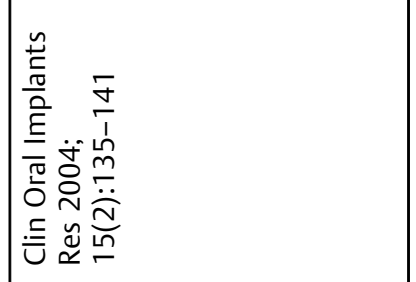 & 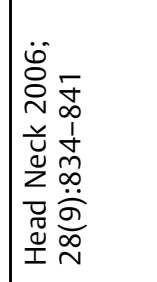 \\
\hline $\begin{array}{l}\text { 高 } \\
\text { 妾 }\end{array}$ & 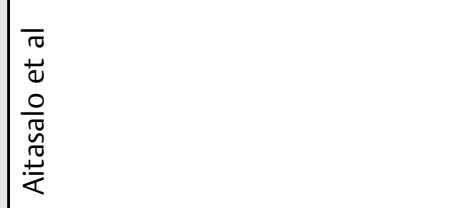 & 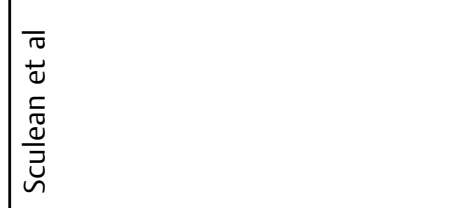 & 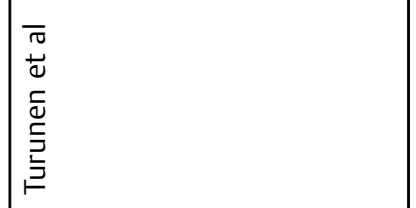 & $\begin{array}{l}\bar{\pi} \\
\frac{\pi}{0} \\
\frac{\pi}{0} \\
\frac{\pi}{0} \\
\alpha\end{array}$ \\
\hline$\stackrel{\varrho}{\Xi}$ & 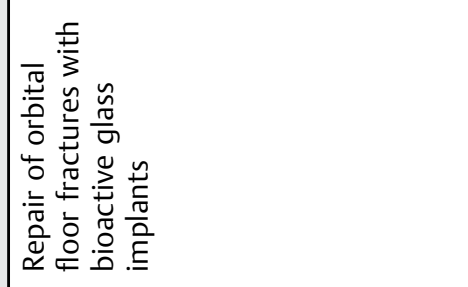 & 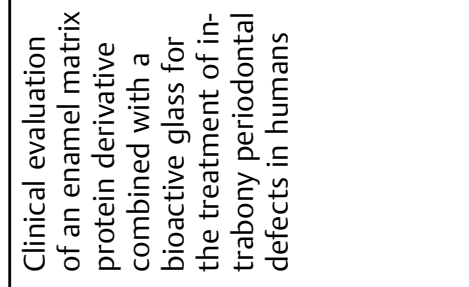 & 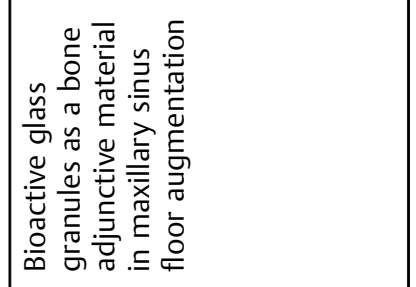 & 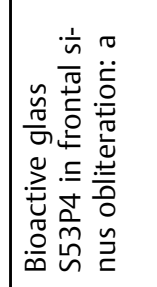 \\
\hline
\end{tabular}




\begin{tabular}{|c|c|c|c|c|}
\hline 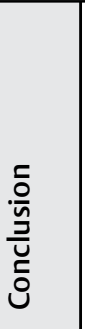 & 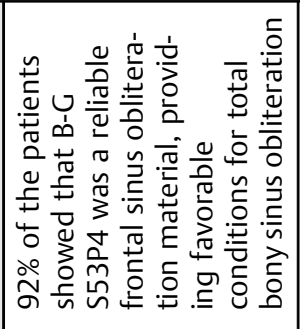 & 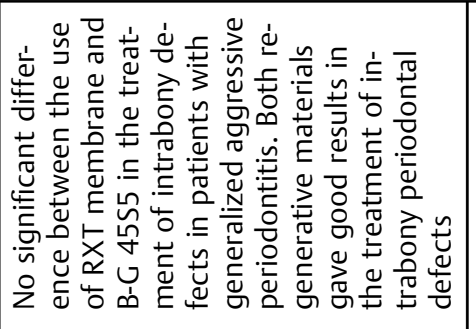 & 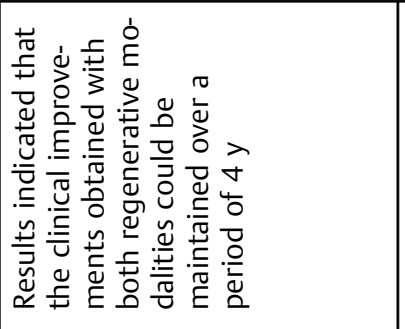 & 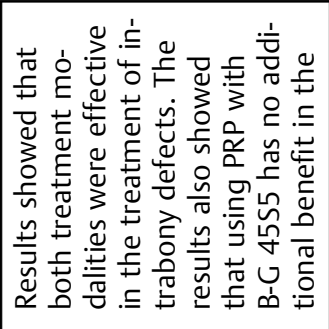 \\
\hline 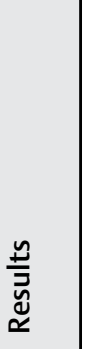 & 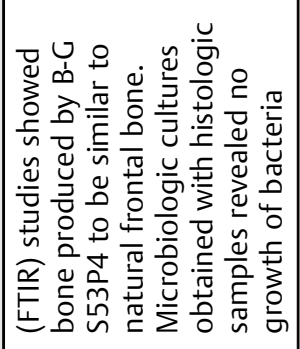 & 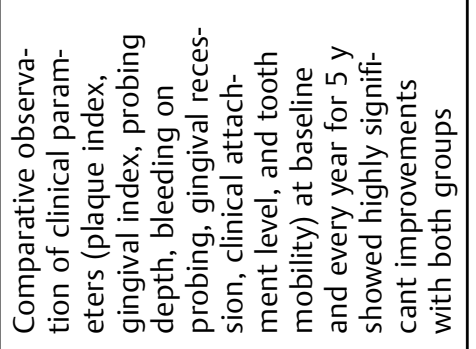 & 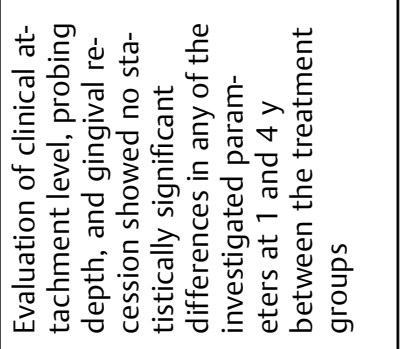 & 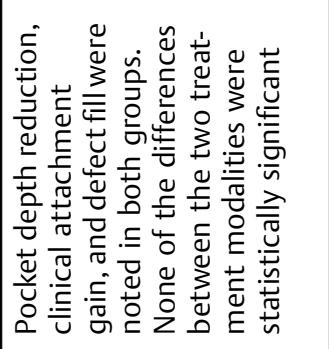 \\
\hline 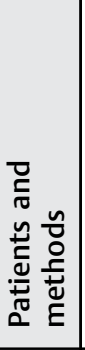 & 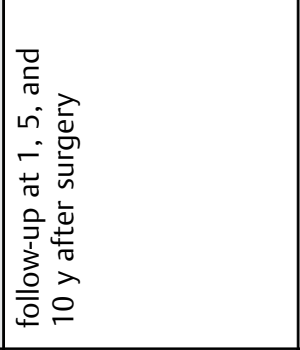 & 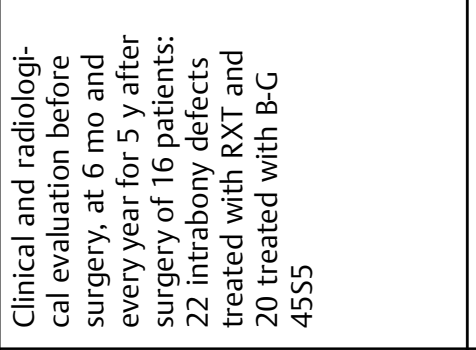 & 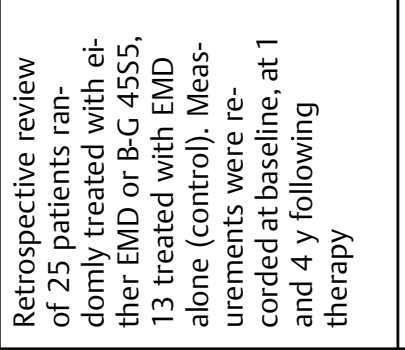 & 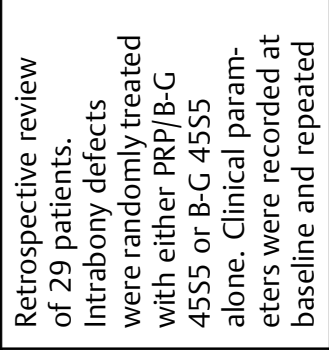 \\
\hline 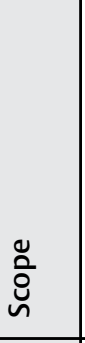 & 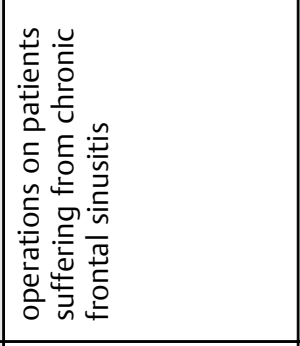 & 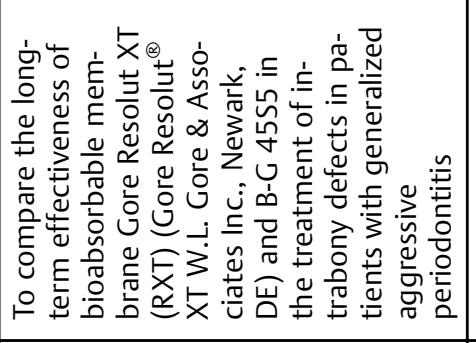 & 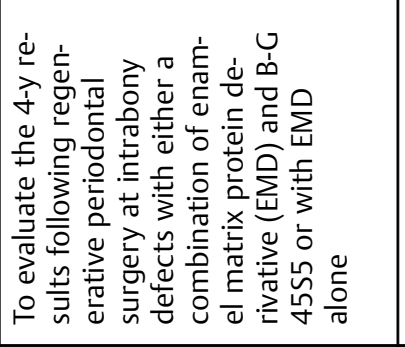 & 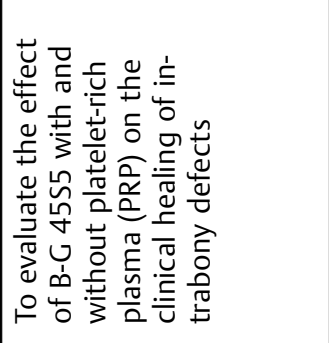 \\
\hline 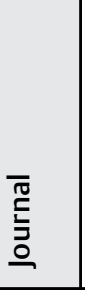 & & 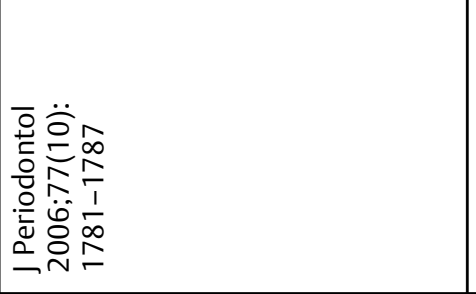 & 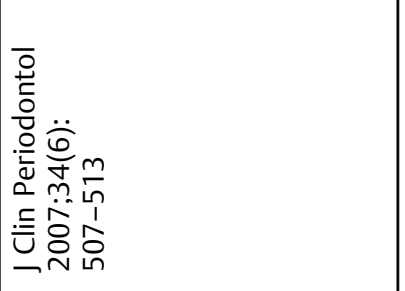 & 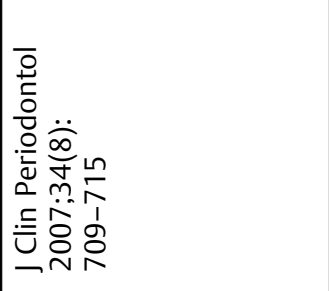 \\
\hline 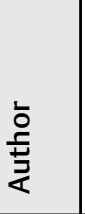 & & $\begin{array}{l}\bar{\pi} \\
\frac{\pi}{\omega} \\
\bar{\Phi} \\
\bar{\sigma} \\
\frac{0}{2}\end{array}$ & $\begin{array}{l}\bar{\pi} \\
\frac{\pi}{\Delta} \\
\frac{\pi}{\pi} \\
\frac{U}{\vec{J}} \\
\sim\end{array}$ & 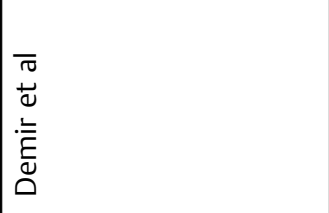 \\
\hline 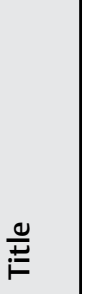 & 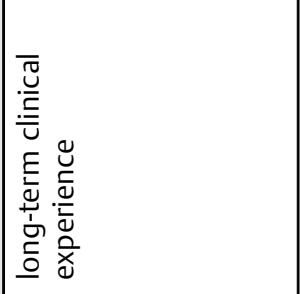 & 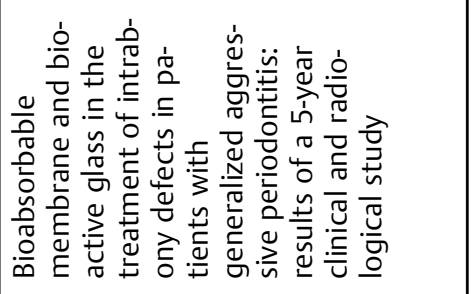 & 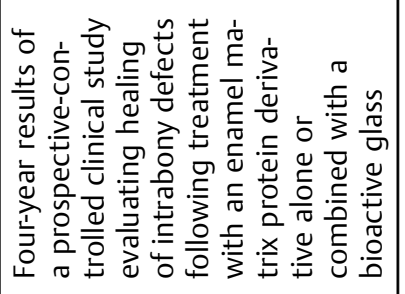 & 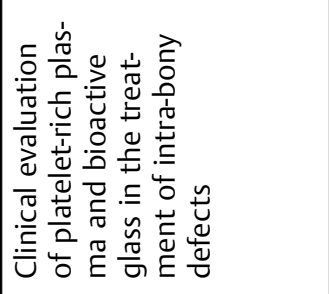 \\
\hline
\end{tabular}




\begin{tabular}{|c|c|c|c|c|}
\hline 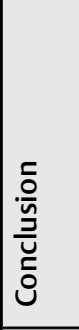 & 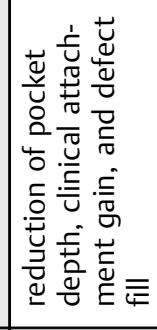 & 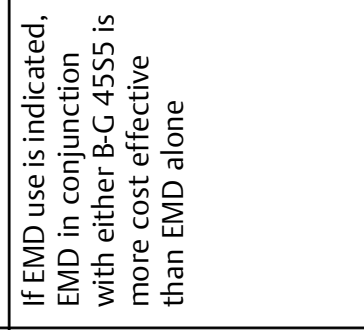 & 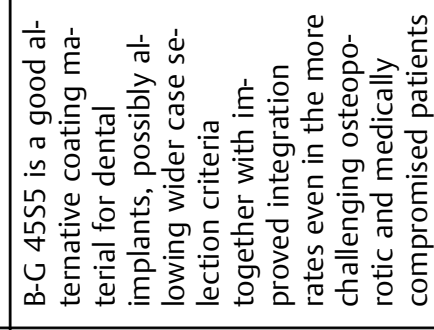 & 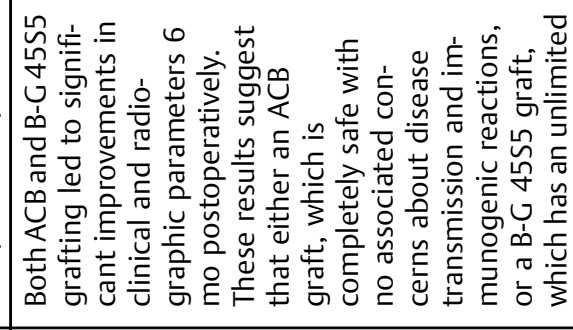 \\
\hline $\begin{array}{l}\frac{n}{\bar{z}} \\
\underline{\underline{u}}\end{array}$ & & 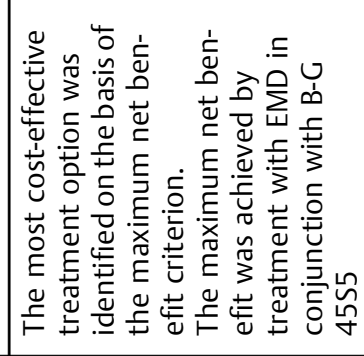 & 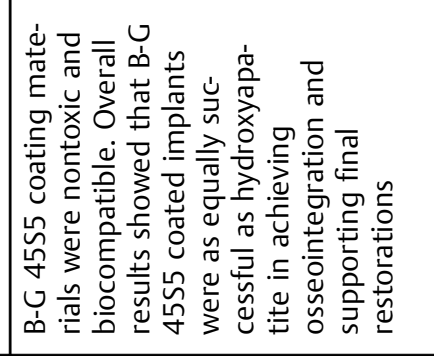 & 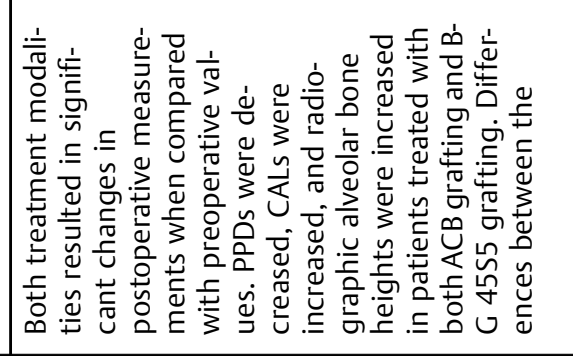 \\
\hline 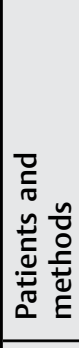 & 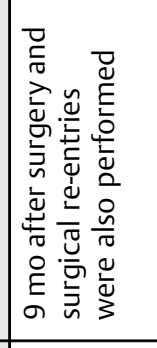 & 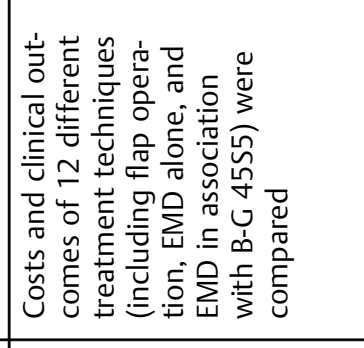 & 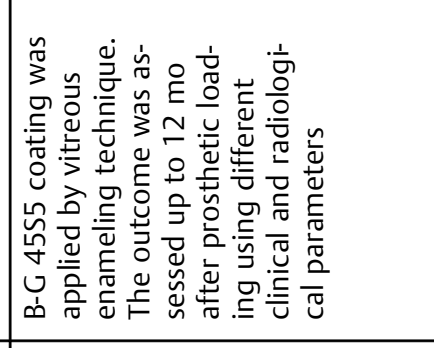 & 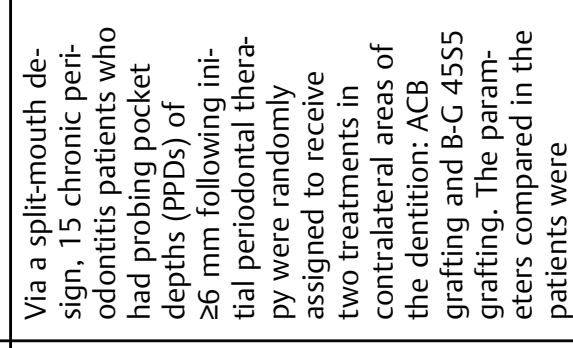 \\
\hline 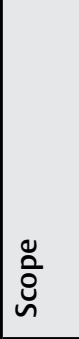 & & 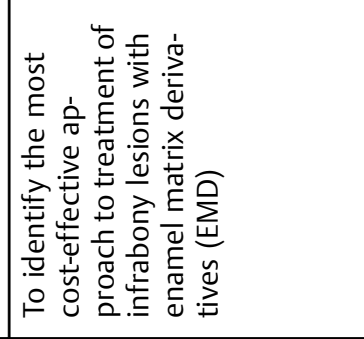 & 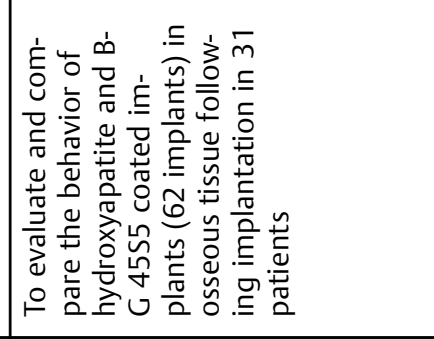 & 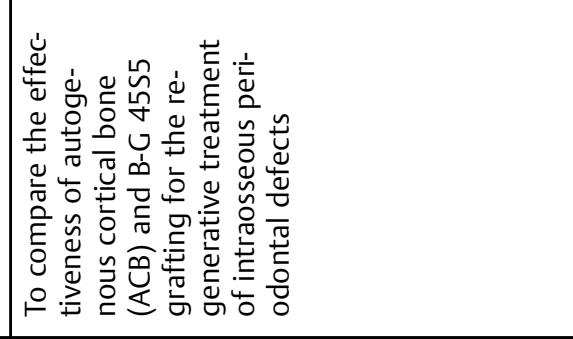 \\
\hline 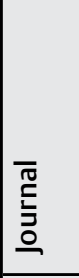 & & 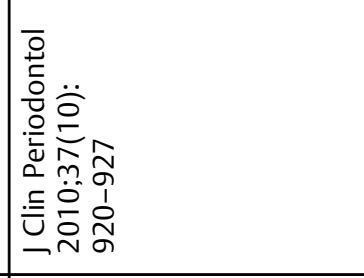 & 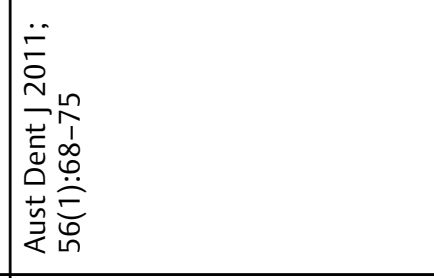 & 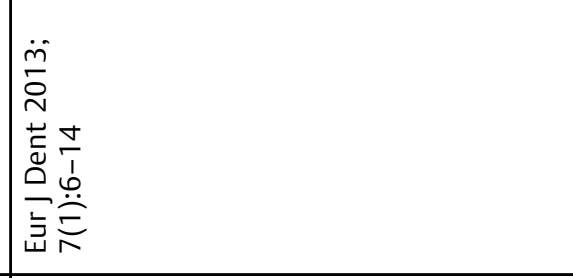 \\
\hline 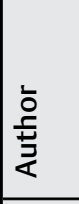 & & $\mid$\begin{tabular}{|l}
$\bar{\pi}$ \\
$\underline{\underline{0}}$ \\
$\underline{\underline{\underline{\underline{y}}}}$
\end{tabular} & 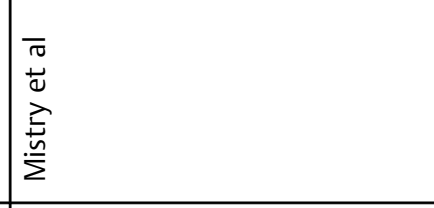 & 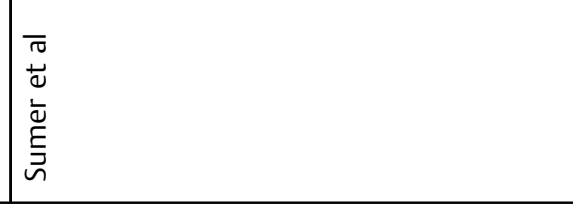 \\
\hline$\stackrel{\varrho}{\rightleftarrows !}$ & & 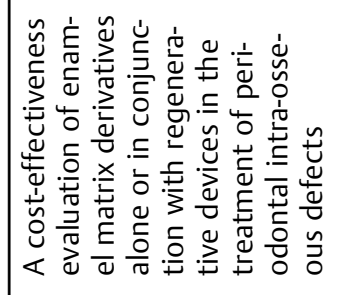 & 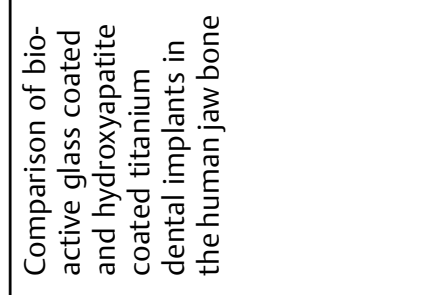 & 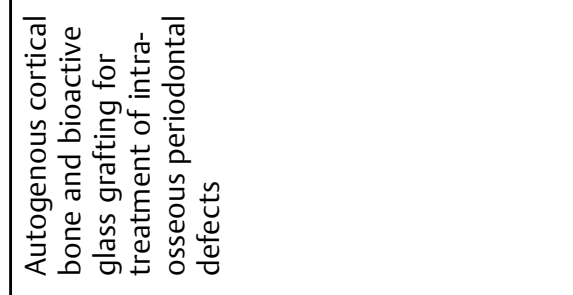 \\
\hline
\end{tabular}




\begin{tabular}{|c|c|c|}
\hline 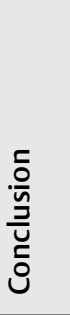 & 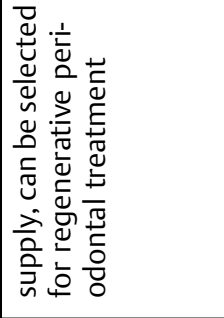 & 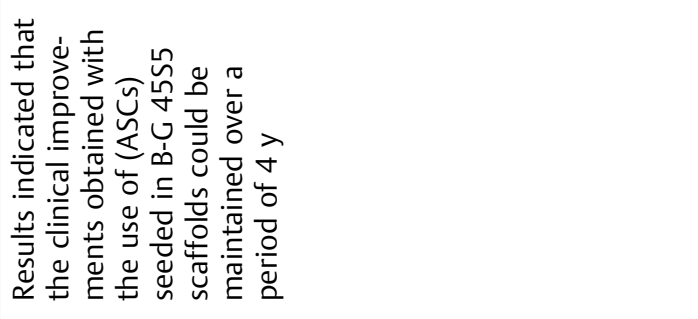 \\
\hline 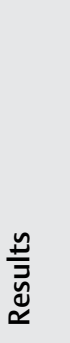 & 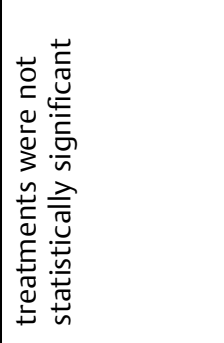 & 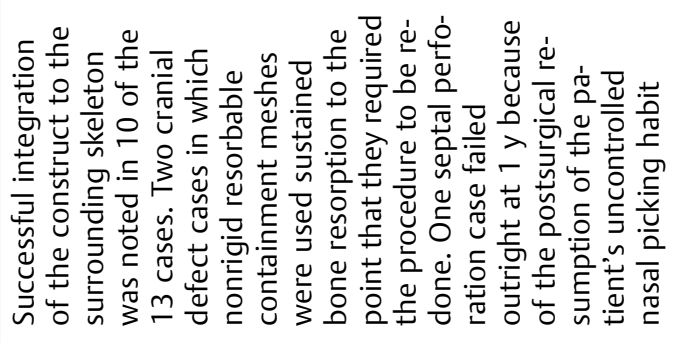 \\
\hline 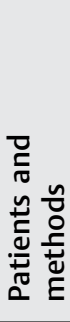 & 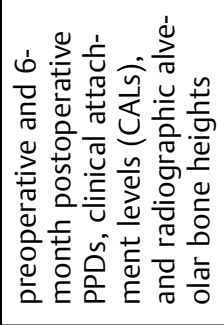 & 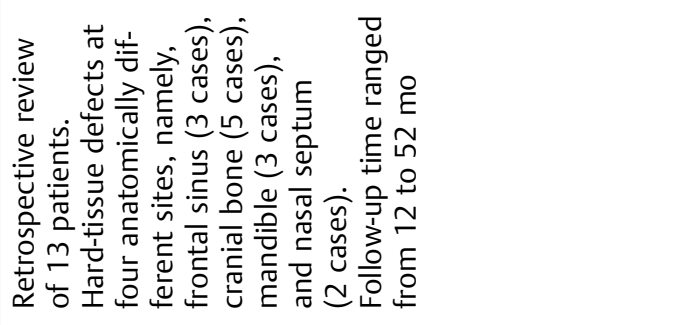 \\
\hline $\begin{array}{l}\text { ڤ̆ } \\
\text { ڤ̆ }\end{array}$ & & 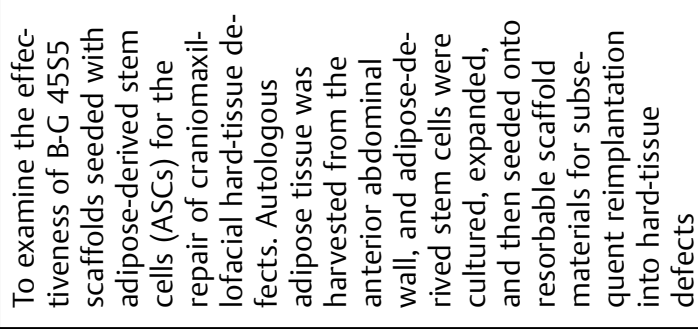 \\
\hline 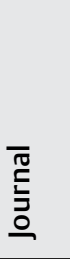 & & 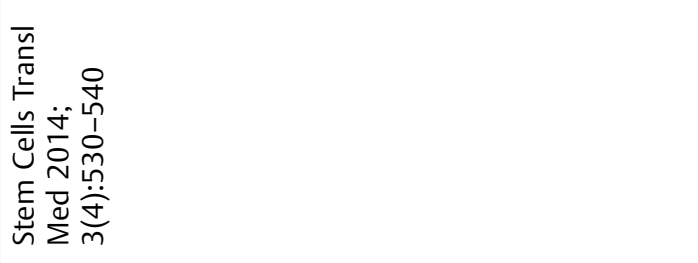 \\
\hline 高 & & 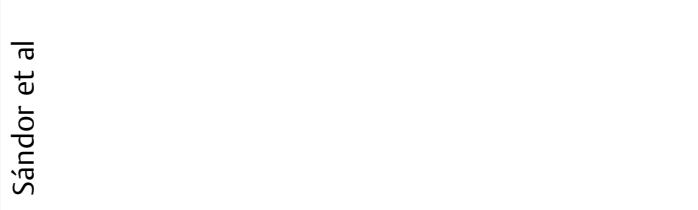 \\
\hline$\stackrel{\Delta}{\underline{I}}$ & & 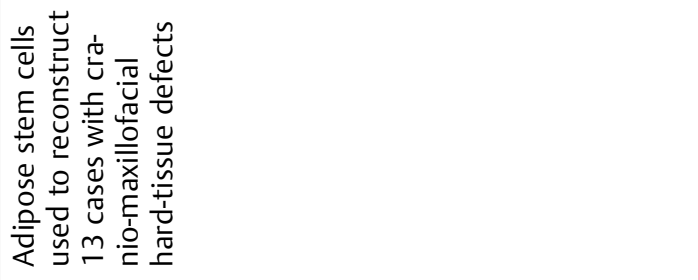 \\
\hline
\end{tabular}



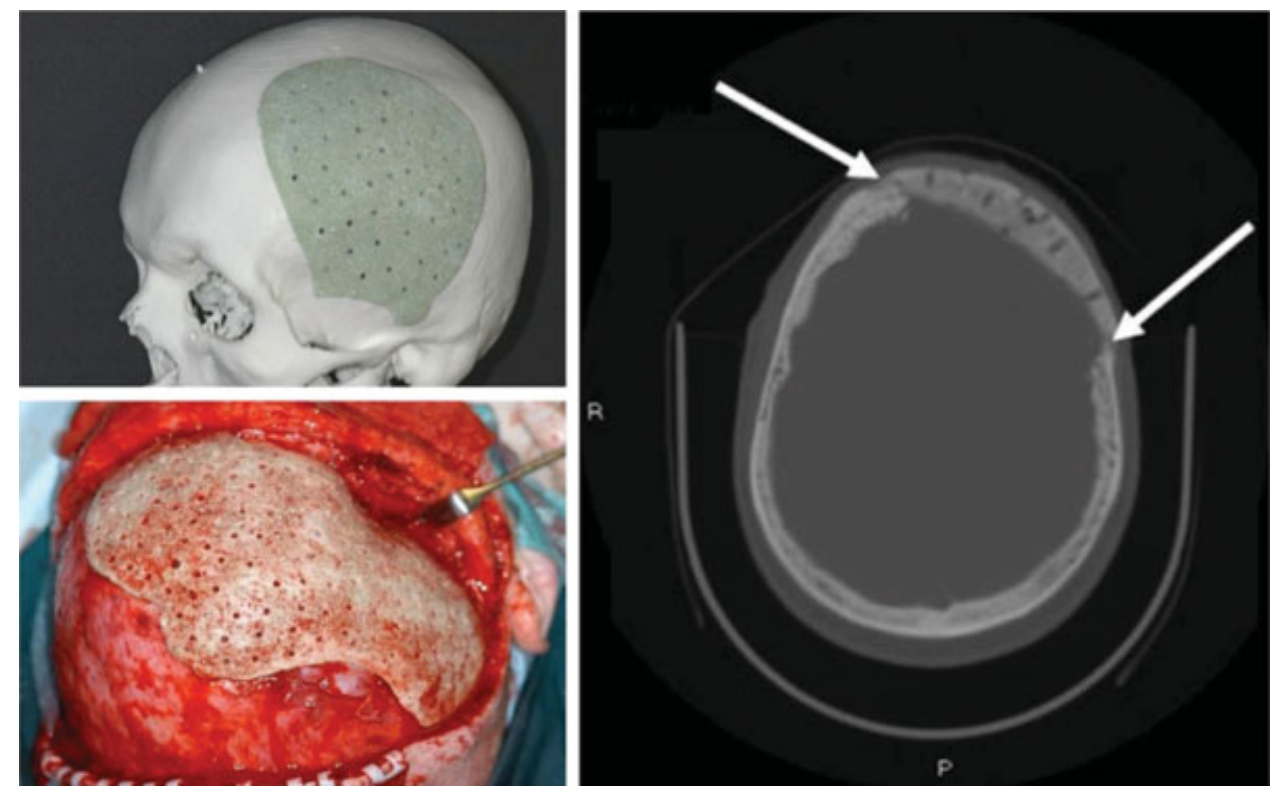

Fig. 2 Additive manufacturing model of a large left calvarial bone defect with a tailor-made PMMA and B-G (S53P4) implant before operation (above, left). Intraoperative picture of a bioactive composite implant adjusted to its correct position in the calvarial bone defect. Note the $1.5 \mathrm{~mm}$ perforations to enhance tissue growth into the alloplastic material (below, left). CT scan of a left temporal bone defect 2 years after reconstruction (right). Implant is in the correct position in the skull. New bone formation between implant and surrounding bone is seen (white arrows). CT, computed tomography; PMMA, polymethylmethacrylate. (Adapted from Peltola et al. ${ }^{30}$ )

toxicities systemically. Readers should also be aware that there was no donor-site morbidity because the custom-made implants did not need any graft operations. The perforated structure of the implants favored tissue ingrowth, yet providing solid flexural modulus and mechanical strength, but the bioactive coating was regarded as the key attribute, especially for those patients with a history of previous reconstruction material removal because of infection. ${ }^{23}$ The strong porous structure of the composite implants mimicked surrounding bone, while S53P4 particles on their surface enabled new hard tissue formation by osteoblasts. The growth and maturation of natural bone in the porous inner layers provided firm adhesion, which in turn prevented longterm complications such as alloplast displacement. Finally, the synthetic implants did not evoke artifacts in postoperative imaging modalities for tumor follow-up. The bioactive coating was permeable to X-rays and performed in CT imaging (-Fig. 2) as well as methacrylate alone..$^{23,30}$ The same applies to magnetic resonance imaging (MRI), as S53P4 is nonmagnetic.

\section{Surgical Approaches to Orbit and Facial Skeleton}

Today, a remarkable share of clinical challenges regarding the fronto-orbital area that require obliterative and reconstructive surgery can be resolved with biomaterials. Such clinical conditions can be chronic suppurative frontal sinusitis, mucoceles, pyoceles, complicated fractures (especially those involving the frontal sinus drainage pathway), and tumors (such as carcinoma). Frontal bone osteomyelitis with bone necrosis and frontal sinus diseases with orbital or intracranial extension after tumor removal are also presumed to be conditions for reconstruction with biomaterials. Defects after subcranial tumor resection in the anterior skull base often need a reconstruction material, and this is another field for the use of tissue replacement materials.

The earlier widely used autogenic tissues are associated with donor-site morbidity, whereas allogenic, animal-derived graft materials and bank tissues have a risk of biohazard infections. ${ }^{34}$ Microvascular free flaps can have aesthetic limitations. Endoscopic Lothrop-type procedures have been recommended to resolve the problems with chronic frontal sinusitis and, recently, even to avoid chronic frontal sinusitis after osteoplastic flap failure. ${ }^{34}$ Despite these advances in frontal sinus surgery, there are still problematic cases where the osteoplastic procedure with obliteration is preferable.

In bone reconstruction with alloplasts, the most important practical factors are an adequate knowledge of their composition along with awareness of specific advantages and disadvantages. Synthetic materials are available in any time and amount, whereas large reconstructions and reoperations may limit the use of autogenic grafts. They are manufactured under controlled conditions, and thus the exact compositions are well known. Another obvious advantage is avoidance of having to harvest autogenic tissues. This decreases the total time needed for surgery, and also the length of wounds is reduced. In experimental studies comparing synthetic biomaterials, B-Gs produced more new bone over the same period than materials such as HA and tricalcium phosphate, ${ }^{35}$ and the bone produced by the first was shown to be more similar to natural frontal bone. HA cement was associated with unsatisfactory long-term results due to exposure of the material, delayed inflammatory reactions, and infections 
originating in the frontal sinus area. ${ }^{36,37}$ Other distinctive qualities of B-Gs are ability to remain where placed even with adjacent suctioning, hemostasis, and incorporation into host bone without the fibrous encapsulation encountered with most other synthetic materials. ${ }^{38}$

Accurate filling with saline liquid-moistened B-G granules (S53P4 formula, $0.5-0.8$ and $0.8-1.0 \mathrm{~mm}$ in size) was used successfully for contour restoration of the facial skeleton and frontal bone reconstruction, ${ }^{39-43}$ with uneventful outcomes or any evidence of resorption. Indeed, the $B-G$ granule remnants seemed to maintain perdurable and uniform bony reconstruction. ${ }^{34}$ When using biologically active materials, it is advisable to know their resorption behavior, and this should closely match the bone formation rate at the reconstruction or implant sites. The slower degradation rate and lack of resorption presented by S53P4 may be due to glass composition, which has higher silica content than the parent 45S5 B-G.

Separate similar studies were performed with tailor-made, rigid B-G plates which were fitted into place for the repair of orbital floor fractures, with good maintenance of globe position during follow-up periods of 5 years, ${ }^{44}$ as well as after fronto-orbital tumor resections, with the only difference that in this case, implants were manufactured casting glass of the S53P4 composition. ${ }^{34,42,43,45}$ Without exception, the biomaterials used were well tolerated, with good functional and aesthetic outcomes (-Figs. 3 and 4). The B-G plate met most of the criteria for a beneficial orbital wall reconstruction material. Besides, it represented the only orbital reconstruc- tion material with antibacterial properties, which may be the reason why acute or late infections were not reported. ${ }^{45}$ This is a very important aspect in orbital wall reconstructions, where a connection between the orbit and the maxillary sinus is common. In fact, infections originating from the maxillary sinus can lead to the need to remove the reconstruction material. ${ }^{45}$ In histologic studies, special attention was paid to new bone formation and conversion of B-G. ${ }^{34}$ The results were in agreement with the findings of other studies, ${ }^{7}$ in which bonding between the glass implant surface and host tissues was formed by attachment of collagen fibers to newly formed silica-rich containing layers. Although B-G is a slowly biodegradable material, the plates were firmly attached to orbital bone structures when histological samples were harvested, indicating immobilization and incorporation to the orbital bone even without specific screw fixation. ${ }^{45}$

\section{Paranasal Sinuses Surgery}

The maxillofacial area is a unique challenge to the surgeon because it is related to infection-sensitive structures such as the paranasal sinuses, upper respiratory tract, and oral cavity. Notably, frequent infections, inflammatory disorders, posttraumatic conditions, and tumors affect the frontal sinus and present unique requirements for successful surgical treatment. Indications for frontal sinus obliteration include problematic conditions that cannot be resolved with functional endoscopic sinus surgery techniques due to irreversibly damaged natural drainage. Such indications can include
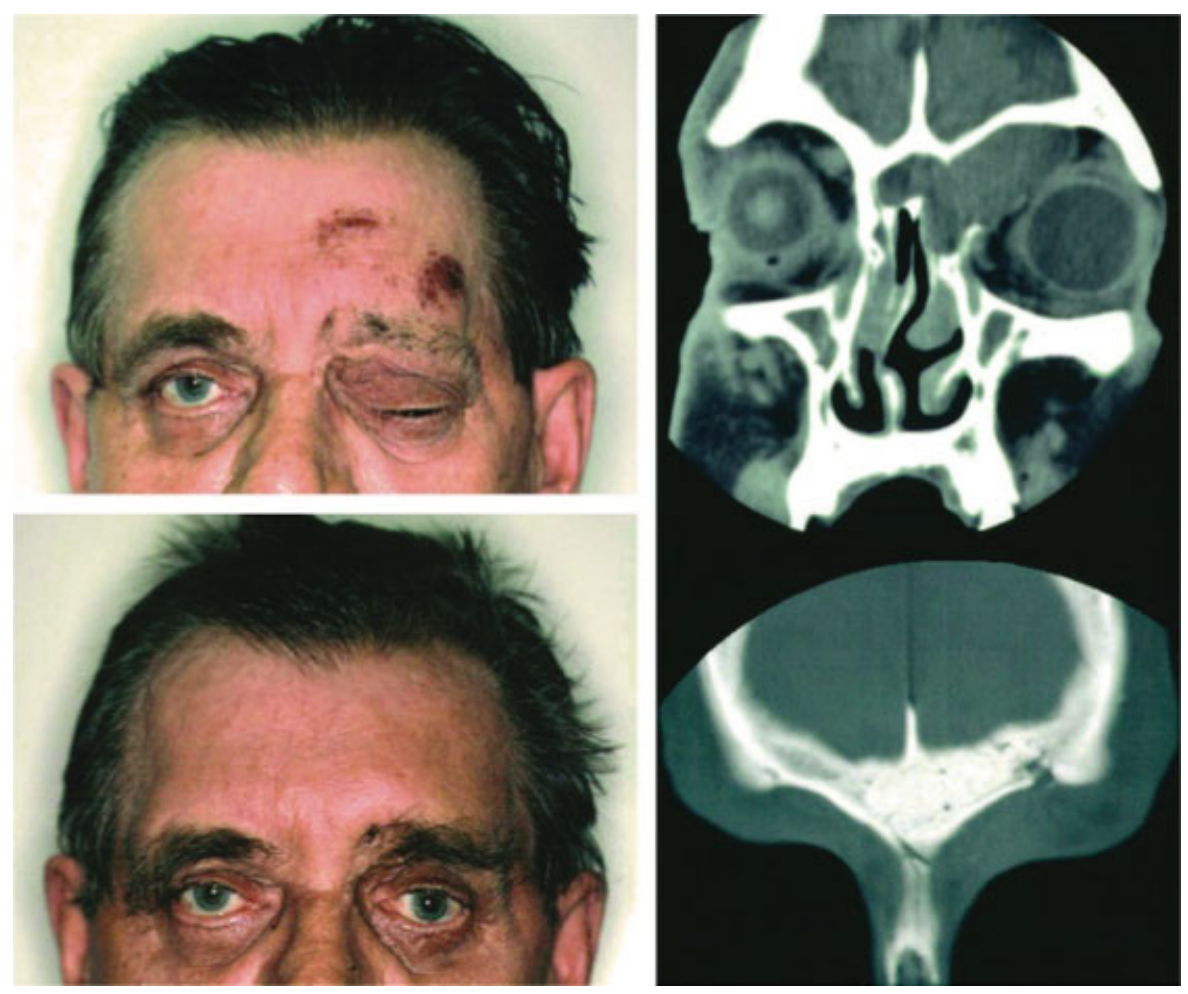

Fig. 3 Three-dimensional CT scan of a complicated fracture of the maxillary sinus and zygomatic arch (left). CT at 1 year after reconstruction of the orbit (above, right) with the B-G implant. Photograph of the patient obtained 3 years after surgery (below, right). (Adapted from Aitasalo and Peltola. ${ }^{34}$ ) 

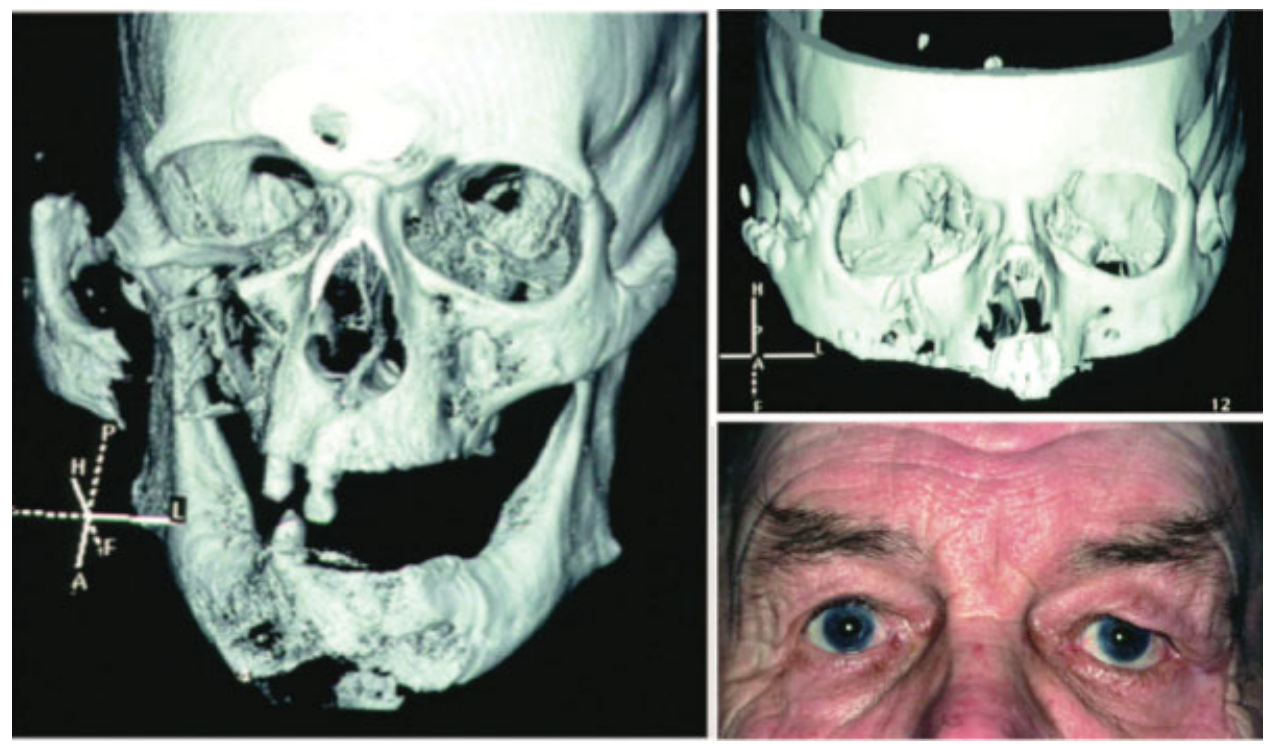

Fig. 4 Mucocele of the left frontal sinus and orbit. Photograph of the patient (above, left) and sinus CT scan in coronal projection (above, right) of the patient before surgery. Photograph of the patient (below, left) and CT scan (below, right) of the patient after frontal sinus obliteration, with $\mathrm{B}-\mathrm{G}$ and calvarial bone transplantation in the roof of the left orbit. (Adapted from Aitasalo and Peltola. ${ }^{34}$ )

chronic suppurative frontal sinusitis, mucoceles, pyoceles, complex fractures (especially those involving the drainage pathway), and tumors, such as osteomas. ${ }^{46}$

Finding a reliable, biocompatible, and safe material for frontal sinus obliteration has proven difficult. Abdominal fat is well established as a versatile and reliable material in frontal sinus obliteration. ${ }^{46}$ In addition, pericranial flaps are interesting alternatives in frontal sinus obliteration. ${ }^{46} \mathrm{Com}-$ pared with HA cement, frontal sinus obliteration with autogenic fat is more cost effective but requires a longer operation time. ${ }^{46}$ However, a risk of donor-site morbidity is related to abdominal fat and autogenic bone transplantation.

Promising results have been described with the use of B-G in chronically infected nasal septum perforations ${ }^{19}$ and after canal wall down mastoidectomy ${ }^{47}$ B-G-ceramic middle ear implants in ossicular chain reconstructions also showed good tolerance after 8 years. ${ }^{18}$ The material appeared to have a broader antimicrobial effect than HA, inhibiting their growth, ${ }^{48}$ and S53P4 glass granules (0.5-0.8 and 0.8$1.0 \mathrm{~mm}$ in diameter) moistened in sterile physiologic saline were subsequently used to obliterate the frontal sinuses with favorable results (- Fig. 3). . $^{31,34,40,46}$ Moistened small granules of B-G are adherent and easy to handle for filling the cavity completely. Hence, a second operation needed for harvesting of autogenous material can be avoided. Repeated CT or MRI scans of frontal sinuses are the only way to accurately monitor the degree of obliteration and identify the patient at risk for recurrent disease after an osteoplastic procedure. A 5-year clinical follow-up study revealed uneventful outcomes, ${ }^{40}$ and bone formation with no loss of volume was reported in the obliterated frontal sinuses over a 10 -year period. ${ }^{31}$ Peltola et $\mathrm{al}^{46}$ characterized the long-term histological healing process in B-G obliterated frontal sinuses. Special attention was given to osteogenesis, occurrence of connective tissue, and possible long-term structural changes in the remaining obliteration materials. The authors demonstrated formation of histologically normal bone without connective tissue in direct contact with the biomaterial particles, in the absence of inflammatory changes or foreign-body reactions. The antibacterial properties of B-G provide extraordinarily favorable conditions and are important factors in obliteration material, that is, in chronic infected frontal sinuses, with a relative risk of reinfection. The predictable healing process and the occlusion of the sinuses decrease the risk of relapse. Laboratory studies showed no abnormal changes in inflammatory parameters or in liver and kidney functions, confirming that B-G is a safe, stable material for permanent clinical frontal sinus obliteration. ${ }^{31}$

B-G particles have also been used to modify the maxillary sinuses. Maxilla grafting for elevation of the maxillary sinus floor with composite grafts of granules of S53P4 glass (80-90 $\mathrm{wt} \%$ ) and autologous bone (10-20 wt\%) was shown to be as good as the treatment with autogenous bone alone, yielding the same quality and volume of mineralized tissue when a reasonable healing period is allowed..$^{33,49-53}$ The use of autogenous bone was dictated by its osteogenic potential related to the number of surviving osteoblasts and osteoinductive effect brought about by the release of bone morphogenic proteins and other growth factors, which have the capacity to accelerate deposition of new bone along the graft material. ${ }^{52}$ Notwithstanding, the amount of bone needed was considerably decreased and donor-site morbidity alleviated. Using histomorphometric analysis, Tadjoedin et $\mathrm{al}^{53}$ found that the composite B-G mixture accelerated healing time for bone regeneration to 6 months, compared with 12 months for bone graft alone. Both treatment regimens resulted in stable bone at the reconstructed site. Accelerated bone healing for elevation of the maxillary sinus floor can allow for simultaneous bone augmentation of the sinus floor and placement of titanium implants for dental restoration in patients who 
would otherwise have insufficient maxillary bone for implant placement. Finally, this combination of findings provides further support for the conceptual premise that the antimicrobial activity of B-G against sinus pathogens ${ }^{31,48}$ might contribute to the resolution of inflammatory responses and provide extraordinarily favorable conditions for an uneventful healing process. ${ }^{49,53}$

\section{Conclusion}

Acting as a resorbable framework in which bone cells can grow, ${ }^{54,55}$ biologically active glasses are an important consideration when choosing the optimal biomaterial to be used as a bone substitute in craniomaxillofacial applications. Their bioactive properties allow for an osteoproductive environment in which the bone-biomaterial interface is uniquely stronger than it would be with other forms of alloplastic materials.

A review of the present literature supports clinical applications of prefabricated implants made of B-G ceramics, as has been used in calvarial and midface bone reconstructions as well as for the repair of orbital floor fractures. Also, present experience with $B-G$ in particulate form, preferably mixed with small amount of autogenous bone, support the convenience of this biomaterial over alternate forms of synthetic graft materials. This protocol has been highly successful for elevation of the maxillary sinus floor in preparation for titanium implant placement in the atrophic maxilla. A similar protocol may prove useful in reconstruction of other areas of the head and neck.

\section{Disclosure}

The authors deny any financial affiliations (e.g., employment, direct payment, stock holdings, retainers, consultantships, patent licensing arrangements, or honoraria) or involvement with any commercial organization with direct financial interest in the subject or materials discussed in this article. The authors are responsible for the content and writing of this article.

\section{References}

1 Beederman M, Alkureishi LW, Lam S, Warnke P, Reid RR. Exchange hybrid cranioplasty using particulate bone graft and demineralized bone matrix: the best of both worlds. J Craniofac Surg 2014; 25(2):451-454

2 Almaiman M, Al-Bargi HH, Manson P. Complication of anterior iliac bone graft harvesting in 372 adult patients from May 2006 to May 2011 and a literature review. Craniomaxillofac Trauma Reconstr 2013;6(4):257-266

3 Eppley BL. Alloplastic implantation. Plast Reconstr Surg 1999; 104(6):1761-1783, quiz 1784-1785

4 Smeets R, El-Moawen A, Jung O, et al. From bench to application: current practices in tissue engineering and its realisation at maxillofacial units in Germany, Austria and Switzerland. J Craniomaxillofac Surg 2014;42(7):1128-1132

5 Hench LL, Splinter RJ, Allen WC, Greenlee TK. Bonding mechanisms at the interface of ceramic prosthetic materials. J Biomed Mater Res 1971;5(6):117-141
6 Hench LL. The story of Bioglass. J Mater Sci Mater Med 2006; 17(11):967-978

7 Wilson J, Pigott GH, Schoen FJ, Hench LL. Toxicology and biocompatibility of bioglasses. J Biomed Mater Res 1981;15(6):805-817

8 Stoor P, Söderling E, Grenman R. Interactions between the bioactive glass S53P4 and the atrophic rhinitis-associated microorganism Klebsiella ozaenae. J Biomed Mater Res 1999;48(6):869-874

9 Day RM. Bioactive glass stimulates the secretion of angiogenic growth factors and angiogenesis in vitro. Tissue Eng 2005;11(5-6): 768-777

10 Leppäranta $\mathrm{O}$, Vaahtio M, Peltola T, et al. Antibacterial effect of bioactive glasses on clinically important anaerobic bacteria in vitro. J Mater Sci Mater Med 2008;19(2):547-551

11 Munukka E, Leppäranta O, Korkeamäki M, et al. Bactericidal effects of bioactive glasses on clinically important aerobic bacteria. J Mater Sci Mater Med 2008;19(1):27-32

12 Rahaman MN, Day DE, Bal BS, et al. Bioactive glass in tissue engineering. Acta Biomater 2011;7(6):2355-2373

13 Lindfors NC, Heikkilä JT, Koski I, Mattila K, Aho AJ. Bioactive glass and autogenous bone as bone graft substitutes in benign bone tumors. J Biomed Mater Res B Appl Biomater 2009;90(1):131-136

14 McAndrew J, Efrimescu C, Sheehan E, Niall D. Through the looking glass; bioactive glass S53P4 (BonAlive $\AA$ ) in the treatment of chronic osteomyelitis. Ir J Med Sci 2013;182(3):509-511

15 Pernaa K, Koski I, Mattila K, et al. Bioactive glass S53P4 and autograft bone in treatment of depressed tibial plateau fractures - a prospective randomized 11-year follow-up. J Long Term Eff Med Implants 2011;21(2):139-148

16 Sarin J, Grénman R, Aitasalo K, Pulkkinen J. Bioactive glass S53P4 in mastoid obliteration surgery for chronic otitis media and cerebrospinal fluid leakage. Ann Otol Rhinol Laryngol 2012;121(9): 563-569

17 Abramovich S, Hannan SA, Huins CT, et al. Prospective cohort comparison of bioactive glass implants and conchal cartilage in reconstruction of the posterior canal wall during tympanomastoidectomy. Clin Otolaryngol 2008;33(6):553-559

18 Reck R, Störkel S, Meyer A. Bioactive glass-ceramics in middle ear surgery. An 8-year review. Ann N Y Acad Sci 1988;523:100-106

19 Stoor P, Grénman R. Bioactive glass and turbinate flaps in the repair of nasal septal perforations. Ann Otol Rhinol Laryngol 2004; 113(8):655-661

20 Stoor P, Pulkkinen J, Grénman R. Bioactive glass S53P4 in the filling of cavities in the mastoid cell area in surgery for chronic otitis media. Ann Otol Rhinol Laryngol 2010;119(6):377-382

21 Sándor GK, Numminen J, Wolff J, et al. Adipose stem cells used to reconstruct 13 cases with cranio-maxillofacial hard-tissue defects. Stem Cells Transl Med 2014;3(4):530-540

22 Profeta AC. Dentine bonding agents comprising calcium-silicates to support proactive dental care: origins, development and future. Dent Mater J 2014;33(4):443-452

23 Aitasalo KM, Piitulainen JM, Rekola J, Vallittu PK. Craniofacial bone reconstruction with bioactive fiber-reinforced composite implant. Head Neck 2014;36(5):722-728

24 Neovius E, Engstrand T. Craniofacial reconstruction with bone and biomaterials: review over the last 11 years. J Plast Reconstr Aesthet Surg 2010;63(10):1615-1623

25 Winder J, Cooke RS, Gray J, Fannin T, Fegan T. Medical rapid prototyping and 3D CT in the manufacture of custom made cranial titanium plates. J Med Eng Technol 1999;23(1):26-28

26 Ducic Y. Titanium mesh and hydroxyapatite cement cranioplasty: a report of 20 cases. J Oral Maxillofac Surg 2002;60(3):272-276

27 Stelnicki EJ, Ousterhout DK. Prevention of thermal tissue injury induced by the application of polymethylmethacrylate to the calvarium. J Craniofac Surg 1996;7(3):192-195

28 Gosain AK; Plastic Surgery Educational Foundation DATA Committee. Bioactive glass for bone replacement in craniomaxillofacial reconstruction. Plast Reconstr Surg 2004;114(2):590-593 
29 Gosain AK; Plastic Surgery Educational Foundation DATA Committee. Biomaterials for reconstruction of the cranial vault. Plast Reconstr Surg 2005;116(2):663-666

30 Peltola MJ, Vallittu PK, Vuorinen V, Aho AA, Puntala A, Aitasalo KM. Novel composite implant in craniofacial bone reconstruction. Eur Arch Otorhinolaryngol 2012;269(2):623-628

31 Peltola M, Aitasalo K, Suonpää J, Varpula M, Yli-Urpo A. Bioactive glass S53P4 in frontal sinus obliteration: a long-term clinical experience. Head Neck 2006;28(9):834-841

32 Stoor P, Söderling E, Grénman R. Bioactive glass S53P4 in repair of septal perforations and its interactions with the respiratory infectionassociated microorganisms Haemophilus influenzae and Streptococcus pneumoniae. J Biomed Mater Res 2001;58(1):113-120

33 Turunen T, Peltola J, Yli-Urpo A, Happonen RP. Bioactive glass granules as a bone adjunctive material in maxillary sinus floor augmentation. Clin Oral Implants Res 2004;15(2):135-141

34 Aitasalo KM, Peltola MJ. Bioactive glass hydroxyapatite in frontoorbital defect reconstruction. Plast Reconstr Surg 2007;120(7): 1963-1972, discussion 1973-1974

35 Peltola MJ, Aitasalo KM, Suonpää JT, Yli-Urpo A, Laippala PJ, Forsback AP. Frontal sinus and skull bone defect obliteration with three synthetic bioactive materials. A comparative study. J Biomed Mater Res B Appl Biomater 2003;66(1):364-372

36 Moreira-Gonzalez A, Jackson IT, Miyawaki T, Barakat K, DiNick V. Clinical outcome in cranioplasty: critical review in long-term follow-up. J Craniofac Surg 2003;14(2):144-153

37 Verret DJ, Ducic Y, Oxford L, Smith J. Hydroxyapatite cement in craniofacial reconstruction. Otolaryngol Head Neck Surg 2005; 133(6):897-899

38 Shapoff CA, Alexander DC, Clark AE. Clinical use of a bioactive glass particulate in the treatment of human osseous defects. Compend Contin Educ Dent 1997;18(4):352-354, 356, 358 passim

39 Dusková M, Smahel Z, Vohradník M, et al. Bioactive glass-ceramics in facial skeleton contouring. Aesthetic Plast Surg 2002;26(4):274-283

40 Peltola M, Suonpää J, Aitasalo K, Varpula M, Yli-Urpo A, Happonen RP. Obliteration of the frontal sinus cavity with bioactive glass. Head Neck 1998;20(4):315-319

41 Peltola M, Suonpää J, Aitasalo K, et al. Experimental follow-up model for clinical frontal sinus obliteration with bioactive glass (S53P4). Acta Otolaryngol Suppl 2000;543:167-169

42 Kinnunen I, Aitasalo K, Pöllönen M, Varpula M. Reconstruction of orbital floor fractures using bioactive glass. J Craniomaxillofac Surg 2000;28(4):229-234

43 Aitasalo K, Kinnunen I, Palmgren J, Varpula M. Repair of orbital floor fractures with bioactive glass implants. J Oral Maxillofac Surg 2001;59(12):1390-1395, discussion 1395-1396
44 Thompson ID. Clinical applications of bioactive glasses for maxillofacial repair. In: Hench LL, Fenn MB, Jones JR, eds. New Materials and Technologies for Healthcare. Singapore: World Scientific; 2011:77-96

45 Peltola M, Kinnunen I, Aitasalo K. Reconstruction of orbital wall defects with bioactive glass plates. J Oral Maxillofac Surg 2008; 66(4):639-646

46 Peltola MJ, Aitasalo KM, Aho AJ, Tirri T, Suonpää JT. Long-term microscopic and tissue analytical findings for 2 frontal sinus obliteration materials. J Oral Maxillofac Surg 2008;66(8): 1699-1707

47 Della Santina CC, Lee SC. Ceravital reconstruction of canal wall down mastoidectomy: long-term results. Arch Otolaryngol Head Neck Surg 2006;132(6):617-623

48 Stoor P, Söderling E, Salonen JI. Antibacterial effects of a bioactive glass paste on oral microorganisms. Acta Odontol Scand 1998; 56(3):161-165

49 Stavropoulos A, Sima C, Sima A, Nyengaard J, Karring T, Sculean A. Histological evaluation of healing after transalveolar maxillary sinus augmentation with bioglass and autogenous bone. Clin Oral Implants Res 2012;23(1):125-131

50 Rickert D, Slater JJ, Meijer HJ, Vissink A, Raghoebar GM. Maxillary sinus lift with solely autogenous bone compared to a combination of autogenous bone and growth factors or (solely) bone substitutes. A systematic review. Int J Oral Maxillofac Surg 2012;41(2): 160-167

51 Tadjoedin ES, de Lange GL, Lyaruu DM, Kuiper L, Burger EH. High concentrations of bioactive glass material (BioGran) vs. autogenous bone for sinus floor elevation. Clin Oral Implants Res 2002; 13(4):428-436

52 Cordioli G, Mazzocco C, Schepers E, Brugnolo E, Majzoub Z. Maxillary sinus floor augmentation using bioactive glass granules and autogenous bone with simultaneous implant placement. Clinical and histological findings. Clin Oral Implants Res 2001; 12(3):270-278

53 Tadjoedin ES, de Lange GL, Holzmann PJ, Kulper L, Burger EH. Histological observations on biopsies harvested following sinus floor elevation using a bioactive glass material of narrow size range. Clin Oral Implants Res 2000;11(4): 334-344

54 Liu X, Rahaman MN, Fu Q. Bone regeneration in strong porous bioactive glass (13-93) scaffolds with an oriented microstructure implanted in rat calvarial defects. Acta Biomater 2013;9(1): 4889-4898

55 Jones JR. Review of bioactive glass: from Hench to hybrids. Acta Biomater 2013;9(1):4457-4486 